\title{
Electronic and optical properties of boron-doped nanocrystalline diamond films
}

\author{
W. Gajewski, ${ }^{1}$ P. Achatz, ${ }^{2,3}$ O. A. Williams, ${ }^{4}$ K. Haenen, ${ }^{4}$ E. Bustarret ${ }^{2}$ M. Stutzmann, ${ }^{1}$ and J. A. Garrido ${ }^{1}$ \\ ${ }^{1}$ Walter Schottky Institut, TU München, Am Coulombwall 3, 85748 Garching, Germany \\ ${ }^{2}$ Institut Néel, CNRS, Université Joseph Fourier, BP 166, 38042 Grenoble, France \\ ${ }^{3}$ INAC, SPSMS, LaTEQS, CEA, F-38054 Grenoble Cedex 9, France \\ ${ }^{4}$ Institute for Materials Research (IMO), Hasselt University, Belgium \\ and Division IMOMEC, IMEC vzw, Wetenschapspark 1 3590, Belgium
}

(Received 13 May 2008; revised manuscript received 6 November 2008; published 15 January 2009)

\begin{abstract}
We report on the electronic and optical properties of boron-doped nanocrystalline diamond (NCD) thin films grown on quartz substrates by $\mathrm{CH}_{4} / \mathrm{H}_{2}$ plasma chemical vapor deposition. Diamond thin films with a thickness below $350 \mathrm{~nm}$ and with boron concentration ranging from $10^{17}$ to $10^{21} \mathrm{~cm}^{-3}$ have been investigated. UV Raman spectroscopy and atomic force microscopy have been used to assess the quality and morphology of the diamond films. Hall-effect measurements confirmed the expected $p$-type conductivity. At room temperature, the conductivity varies from $1.5 \times 10^{-8} \Omega^{-1} \mathrm{~cm}^{-1}$ for a nonintentionally doped film up to $76 \Omega^{-1} \mathrm{~cm}^{-1}$ for a heavily $B$-doped film. Increasing the doping level results in a higher carrier concentration while the mobility decreases from 1.8 down to $0.2 \mathrm{~cm}^{2} \mathrm{~V}^{-1} \mathrm{~s}^{-1}$. For NCD films with low boron concentration, the conductivity strongly depends on temperature. However, the conductivity and the carrier concentration are no longer temperature dependent for films with the highest boron doping and the NCD films exhibit metallic properties. Highly doped films show superconducting properties with critical temperatures up to $2 \mathrm{~K}$. The critical boron concentration for the metal-insulator transition is in the range from $2 \times 10^{20}$ up to $3 \times 10^{20} \mathrm{~cm}^{-3}$. We discuss different transport mechanisms to explain the influence of the grain boundaries and boron doping on the electronic properties of NCD films. Valence-band transport dominates at low boron concentration and high temperatures, whereas hopping between boron acceptors is the dominant transport mechanism for borondoping concentration close to the Mott transition. Grain boundaries strongly reduce the mobility for low and very high doping levels. However, at intermediate doping levels where hopping transport is important, grain boundaries have a less pronounced effect on the mobility. The influence of boron and the effect of grain boundaries on the optoelectronic properties of the NCD films are examined using spectrally resolved photocurrent measurements and photothermal deflection spectroscopy. Major differences occur in the low energy range, between 0.5 and $1.0 \mathrm{eV}$, where both boron impurities and the $s p^{2}$ carbon phase in the grain boundaries govern the optical absorption.
\end{abstract}

DOI: 10.1103/PhysRevB.79.045206

PACS number(s): 72.20.-i, 78.20.-e, 78.30.-j

\section{INTRODUCTION}

Nanocrystalline diamond (NCD) thin films have, among other outstanding properties, a remarkable hardness, low friction coefficient, optical transparency in the ultraviolet, visible, and infrared, as well as a low electron emission threshold voltage. Different applications have been so far reported in which NCD films were used for high-frequency surface acoustic wave devices, ${ }^{1,2}$ microelectromechanical systems (MEMSs), ${ }^{3}$ metal-semiconductor field-effect transistors (MESFETs), ${ }^{4}$ and enduring coating. ${ }^{5,6}$ Due to its biocompability and chemical stability, NCD films have also attracted attention for bioelectrochemical applications. ${ }^{7-9}$ In addition, considerable efforts have been dedicated to optimize the growth process: its dependence on gas preparation and precursor molecules, ${ }^{10,11}$ as well as the influence of the substrate material. ${ }^{12,13}$ The growth and structural properties of NCD films and ultrananocrystalline diamond (UNCD) films have been recently compared. ${ }^{14}$ So far, the $n$-type conductivity induced by nitrogen addition during UNCD growth has been investigated in detail. ${ }^{15,16}$ The $p$-type doping of diamond with boron has been extensively examined in single-crystal [single-crystal diamond (SCD)] homoepitaxially grown material, ${ }^{17-20}$ as well as in polycrystalline [poly- crystalline diamond (PCD)] materials..$^{21,22}$ In the case of heavily boron-doped samples, superconductivity has been reported. ${ }^{23,24}$ Finally, the influence of the boron acceptor on the structural and optoelectronic properties of the diamond films was investigated for single and polycrystalline diamond films. ${ }^{21,22,25-30}$

However, the same sort of detailed investigation is still missing for boron-doped NCD films. As it has been discussed for polycrystalline diamond films ${ }^{22}$ and UNCD films,${ }^{31}$ it is essential to understand the influence of the grain boundaries on the optical properties of NCD and the role they play in the electronic transport. Recently, several papers have reported on the structural properties of NCD films, as revealed by Raman spectroscopy in low $^{32-34}$ and in high boron-doped diamond films. ${ }^{34-37}$ The characteristic Fano resonance behavior was observed for heavily doped NCD films. ${ }^{38}$ However, a comprehensive study on the electronic properties has not yet been published. Some data can be found in articles focused on the NCD structure ${ }^{36,39}$ or in a recently published work on the superconductivity of borondoped nanocrystalline diamond. ${ }^{38}$

In this work we have systematically investigated the structural, electronic, and optical properties of NCD films with boron concentrations from $10^{16}$ up to $10^{21} \mathrm{~cm}^{-3}$. Ultraviolet micro-Raman spectroscopy was used to study the 
structural properties of the NCD films. Temperaturedependent Hall-effect experiments and conductivity experiments were applied to investigate the influence of boron on the electronic properties of NCD films. Finally, UV-vis spectroscopy, spectrally resolved photoconductivity, and photothermal deflection spectroscopy were used to study the optical properties and the influence of the grain boundaries.

The experiments reveal that the conductivity can be well controlled by the boron content in the gas phase, resulting in NCD film behaviors ranging from insulating to metallic. Different electronic transport mechanisms can be observed depending on the concentration of boron in NCD films. While transport in the valence band is expected at high temperature in the nonintentionally doped (NID) and weakly borondoped films, metal-like conductivity dominates in films with boron concentration above $2 \times 10^{20} \mathrm{~cm}^{-3}$. For intermediate boron doping $\left(2 \times 10^{19}-3 \times 10^{20} \mathrm{~cm}^{-3}\right)$, hopping between boron acceptors is assumed to be the dominant transport mechanism in the NCD films. At low temperatures and low boron concentration, hopping transport in the grain boundaries is expected to become important. In other polycrystalline materials, such as microcrystalline and nanocrystalline $\mathrm{Si}$, electronic transport is strongly influenced by the potential barriers at the grain boundaries, as demonstrated by Seto. ${ }^{40}$ We have also addressed this possibility in the case of NCD. However, in contrast to $\mathrm{Si}$, we have found that the effect of grain boundaries is much lower for nanocrystalline diamond due to the large activation energy of the boron acceptor in diamond. Highly doped films show superconducting properties with critical temperatures of about $2 \mathrm{~K}$. A dopinginduced metal-insulator transition is observed, with critical boron concentrations in the range from $2 \times 10^{20}$ up to 3 $\times 10^{20} \mathrm{~cm}^{-3}$. The electronic transport data are in good agreement with the results of the photocurrent experiments. The boron incorporation results in an increase in the film conductivity and a simultaneous photocurrent increase in the low energy range $(0.5-1.0 \mathrm{eV})$. The photocurrent onset observed for the boron-free and NID NCD films at about 1.0 $\mathrm{eV}$ is tentatively assigned to the contribution of $s p^{2}$ bonded carbon in the grain boundaries. The optical-absorption coefficient estimated from photothermal deflection spectroscopy is in good agreement with the results of the photocurrent experiments, confirming the dominant role of the $s p^{2}$ phase on the optical properties of NCD.

\section{EXPERIMENTAL}

Nanocrystalline diamond films with different boron concentrations were grown on quartz substrates. The quartz substrates were seeded ultrasonically in a solution containing nanodiamond powder ${ }^{41}$ and films were grown in a microwave plasma enhanced chemical vapor deposition (MWPECVD) reactor using conventional $\mathrm{CH}_{4} / \mathrm{H}_{2}$ plasma with a methane concentration below 5\%. The boron doping was controlled by adding TriMethylBoron (TMB) to the gas phase, with $\mathrm{B}: \mathrm{C}$ ratios up to $6600 \mathrm{ppm}$.

Prior to any characterization, "as-grown" films were cleaned in ultrasonic baths of acetone and isopropanol. Raman spectroscopy was performed at ambient conditions us- ing a Dilor XY double monochromator spectrometer together with an $\mathrm{Ar}^{+}$ion laser (514.5 nm line) as well as the Dilor LabRam infinity with a $325 \mathrm{~nm}$ line of the HeCd laser. The surface morphology was examined with a Veeco Nanoscope III atomic force microscopy (AFM) in tapping mode under ambient conditions. In order to avoid the influence of the $\mathrm{H}$-induced surface conductivity on the electronic transport experiments, the NCD films were oxidized using oxygen plasma (5 min, $200 \mathrm{~W}, 1.4 \mathrm{mbar})$. Ti/Au (20/200 nm) metal contacts were evaporated in a van der Pauw configuration to investigate the electronic properties of the NCD films. Conductivity and Hall-effect measurements were performed using an Oxford cryostat in the temperature range from 70 to $610 \mathrm{~K}$. For the Hall-effect characterization, an alternating magnetic field of $1.8 \mathrm{~T}$ was used. The temperature dependence of the conductivity in the so-called low-temperature regime, down to $350 \mathrm{mK}$, was investigated using a Quantum Design physical properties measurements system, employing a four-terminal configuration for the contacts. The optical properties were investigated using spectrally resolved photoconductivity (SPC) and photothermal deflection spectroscopy (PDS). In both cases chopped $(4 \mathrm{~Hz})$ monochromatic lights from either a halogen or xenon arc lamp were used for the optical excitation in the energy range of $0.3-6.2 \mathrm{eV}$. The photoresponse was detected by a lock-in amplifier and normalized to the number of incident photons measured by a pyroelectric detector. The photoconductivity measurements were carried out in vacuum $\left(10^{-6}\right.$ mbar $)$ in the temperature range of $80-360 \mathrm{~K}$ using a liquid nitrogen cryostat. The applied bias to the samples during the experiments was in the range of 20-35 V. The PDS measurements were performed at room temperature using perfluorhexane as the deflection medium.

Secondary ion mass spectroscopy was performed by the Centre for Surface and Materials Analysis (CSMA Ltd.) in England using a Cameca IMS 4f double focusing secondary ion mass spectrometer with $12.5 \mathrm{kV} \mathrm{O}_{2}^{+}$primary beam. Under incident angle of $30^{\circ}$ to normal with a nominal impact energy of $8 \mathrm{kV}$ per $\mathrm{O}_{2}{ }^{+}$ion, the sputtering was about $5 \AA / \mathrm{s}$. Calibration was performed using a $5 \times 10^{14} \mathrm{~cm}^{-2}{ }^{11} \mathrm{~B}$ ion implanted diamondlike carbon standard.

\section{RESULTS AND DISCUSSION}

\section{A. Structural properties}

As derived from secondary-ion-mass spectroscopy (SIMS) measurements, the boron concentration in the NCD films varies from $9.7 \times 10^{16} \mathrm{~cm}^{-3}$ in the NID film up to $3.3 \times 10^{21} \mathrm{~cm}^{-3}$ in the heavily boron-doped NCD film (Table I). The SIMS profiles for B-doped NCD films are depicted in Fig. 1. The presence of boron in the NID film results from the memory effect (due to boron contamination) in the growth chamber. A reference film (S1) was grown in a boron-free reactor. It was confirmed that the method applied for seeding has an important impact on the quality of the NCD films ${ }^{14}$ and thus, the efficiency of doping can be increased: see for instance the properties of samples S4, seeded mechanically and with $150 \mathrm{ppm}$ of TMB in the gas phase, and S5, seeded in ultrasonic bath with $10 \mathrm{ppm}$ of TMB in the 
TABLE I. A summary of the main characteristics of NCD films. Samples S1, S2, and S3 (*) did not show the Hall effect.

\begin{tabular}{lccccccc}
\hline \hline Sample & $\begin{array}{c}\text { B/C gas phase } \\
(\mathrm{ppm})\end{array}$ & Seeding $^{\mathrm{a}}$ & $\begin{array}{c}{[\mathrm{B}]_{\text {SIMS }}} \\
\left(\mathrm{cm}^{-3}\right)\end{array}$ & $\begin{array}{c}\sigma\left(\Omega^{-1} \mathrm{~m}^{-1}\right) \\
\text { at } 295 \mathrm{~K}\end{array}$ & $\begin{array}{c}p\left(\mathrm{~cm}^{-3}\right) \\
\text { at } 295 \mathrm{~K}\end{array}$ & $\begin{array}{c}\mu\left(\mathrm{cm}^{2} \mathrm{~V}^{-1} \mathrm{~s}^{-1}\right) \\
\text { at } 295 \mathrm{~K}\end{array}$ & $\begin{array}{c}E_{A} \\
(\mathrm{meV})\end{array}$ \\
\hline S1 & & $\mathrm{m}$ & & below $10^{-10}$ & $*$ & $*$ & 690 \\
S2 & 0 & os & $9.7 \times 10^{16}$ & $1.5 \times 10^{-8}$ & $*$ & $*$ & 390 \\
S3 & 10 & os & $6.0 \times 10^{18}$ & $2.7 \times 10^{-4}$ & $*$ & $*$ & 165 \\
S4 & 150 & m & $2.7 \times 10^{19}$ & $2.4 \times 10^{-3}$ & $7 \times 10^{15}$ & 2.11 & 119 \\
S5 & 10 & os & $2.9 \times 10^{19}$ & $2.2 \times 10^{-2}$ & $1.2 \times 10^{17}$ & 1.12 & 102 \\
S6 & 20 & os & $6.9 \times 10^{19}$ & 0.25 & $6.9 \times 10^{18}$ & 0.22 & 90 \\
S7 & 2500 & m & $2.3 \times 10^{20}$ & 0.81 & $3.9 \times 10^{19}$ & 0.13 & 67 \\
S8 & 1000 & os & $2.9 \times 10^{20}$ & 6.16 & $2.5 \times 10^{20}$ & 0.15 & 27 \\
S9 & 3000 & os & $1.3 \times 10^{21}$ & 29.3 & $1.1 \times 10^{21}$ & 0.16 & 13 \\
S10 & 5000 & m & $1.3 \times 10^{21}$ & 12.1 & $5.6 \times 10^{20}$ & 0.31 & 22 \\
S11 & 4500 & os & $1.8 \times 10^{21}$ & 63.6 & $1.1 \times 10^{21}$ & 0.36 & 12 \\
S12 & 6660 & os & $3.3 \times 10^{21}$ & 75.8 & $2.0 \times 10^{21}$ & 0.24 & 12 \\
\hline \hline
\end{tabular}

as: ultrasonic nanoparticle seeding, m: mechanical scratching technique.

gas phase. However, the influence of the chamber memory effect on the final effective boron doping of the NCD films cannot be excluded. Further measurements should be performed in order to clarify the dependence of the effective boron doping on TMB concentration in the gas phase.

Raman spectroscopy was used to investigate the quality of the diamond films. Typical Raman spectra of the NCD films are presented in Fig. 2 together with the spectra of one undoped single crystalline diamond film and one nitrogen-free UNCD film. The spectra of the NCD films are comparable to those obtained by other authors. ${ }^{32-34}$ Typically, in addition to the first-order diamond peak at $1332 \mathrm{~cm}^{-1}$, the $D$ (expected at $1350 \mathrm{~cm}^{-1}$ ) (Ref. 34) and $G$ (expected at $1600 \mathrm{~cm}^{-1}$ ) (Ref. 34) bands related to $s p^{2}$ carbon are visible due to the contribution of grain boundaries in the NCD material. The position of the first-order diamond peak shifts slightly from

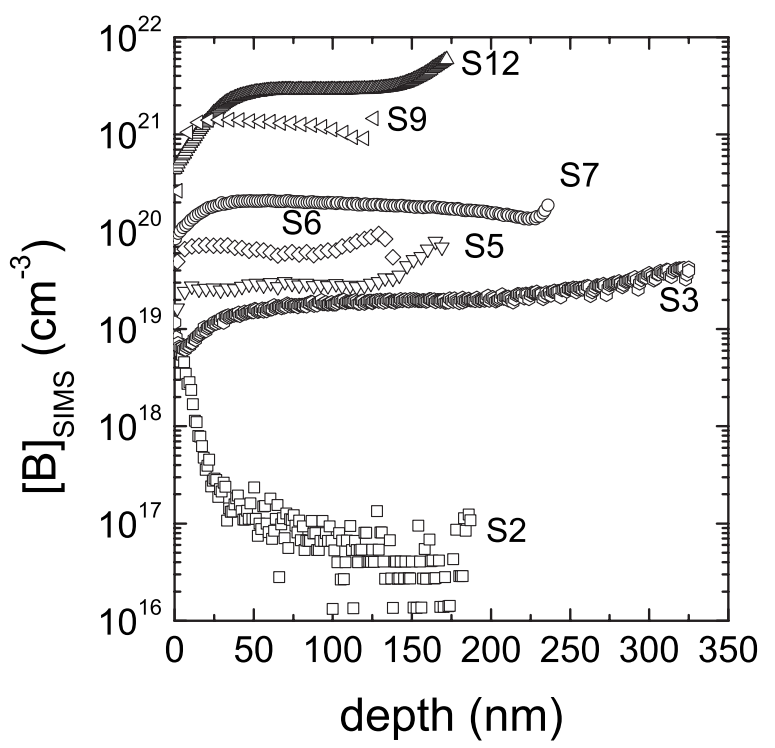

FIG. 1. Doping profiles of boron-doped NCD films as measured by SIMS. the $1332.5 \mathrm{~cm}^{-1}$ position in natural diamond ${ }^{34}$ toward lower wave numbers (see Fig. 3 for details). There are two main reasons which could explain the shift: the influence of doping and the influence of the grain size. The influence of boron doping on the diamond first-order Raman line has been well characterized for single and polycrystalline diamond films, and it has been explained by the Fano-type interference between the continuum of electronic states introduced by the dopants and the discrete zone-center phonon. ${ }^{32-34,42}$

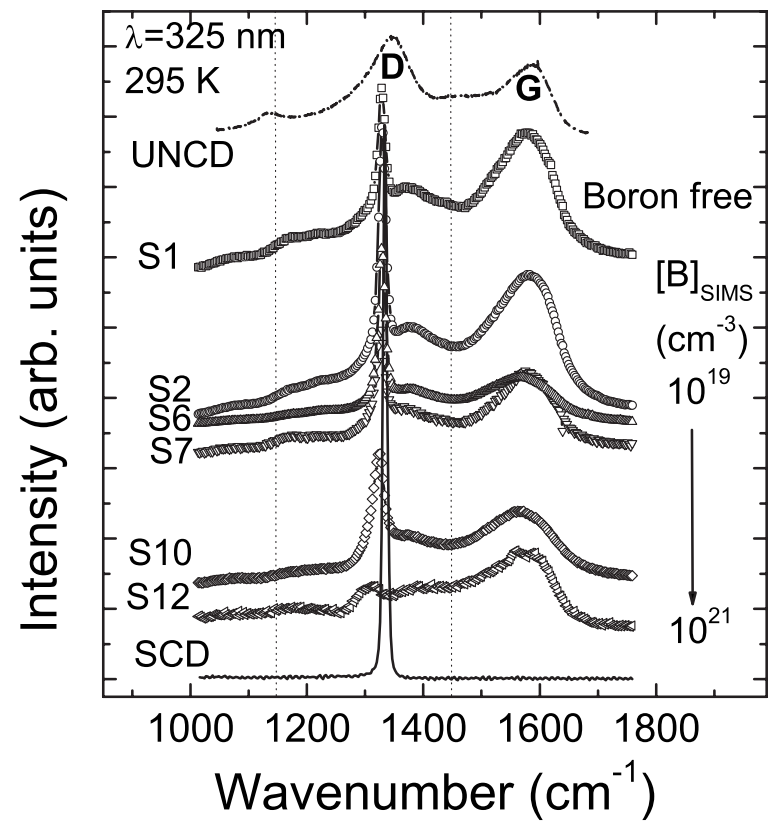

FIG. 2. Raman spectra of boron-doped NCD films compared with Raman spectra of undoped SCD and UNCD films. The main features in the spectra previously reported in NCD films (Refs. 33-35) are marked with the dashed line. Spectra were recorded with $325 \mathrm{~nm}$ excitation line at ambient conditions. The spectra are shifted vertically for clarity. 


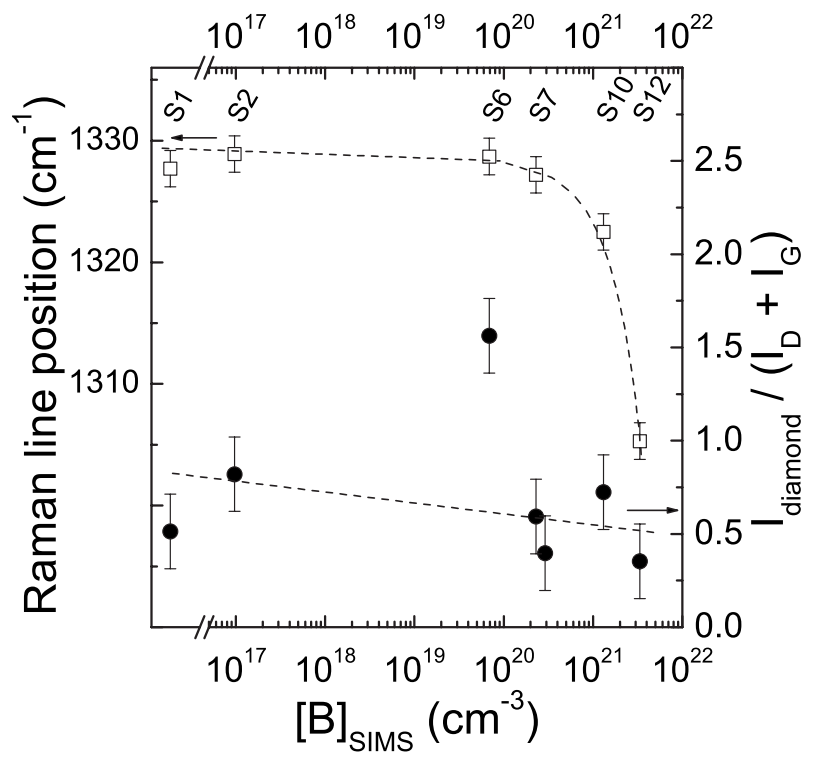

FIG. 3. Position of the diamond first-order line estimated from Raman spectra and the ratio of diamond first-order line $I_{\text {diamond }}$ intensity to the sum of $D$ and $G$ band insensitivities, $I_{D}$ and $I_{G}$, respectively, as a function of boron concentration obtained by SIMS.

The most pronounced effect was observed for films with boron concentration higher than $10^{20} \mathrm{~cm}^{-3}$, which corresponds to the onset of metallic type conductivity for B-doped diamond films. ${ }^{32-34,42}$ Figure 3 depicts the position of the first-order diamond Raman line as a function of the boron concentration obtained by SIMS. A small shift toward lower wave numbers is observed as the doping level increases; the first-order line position for a NID NCD film (S2) is at $1328.9 \mathrm{~cm}^{-1}$. In the case of the most heavily doped NCD film S12 $\left([\mathrm{B}]_{\text {SIMS }}=3.3 \times 10^{21} \mathrm{~cm}^{3}\right)$ the shift is much stronger and the first-order line is located at $1305.3 \mathrm{~cm}^{-1}$. This shift is consistent with the data reported for boron-doped polycrystalline diamond films ${ }^{42}$ and its origin can be attributed to the increasing Fano interference due to the increase in boron content in the NCD films and to the doping-induced lattice expansion. The ratio of the first-order line $I_{\text {diamond }}$ intensity to the sum of $D$ and $G$ band intensities, $I_{D}$ and $I_{G}$, respectively, is also plotted in Fig. 3. Lower values are observed for samples with higher boron concentration, which is consistent with the data reported for polycrystalline diamond films, where a decrease in first-order diamond line intensity as a function of boron doping was observed. ${ }^{34,42}$ It is worth to mention that a rather constant value of the $I_{\text {diamond }} /\left(I_{D}\right.$ $\left.+I_{G}\right)$ ratio with boron concentration would suggest a negligible influence of the doping on the structure and thus on the quality of the boron-doped NCD films.

A typical Fano-type line shape accompanied by a band emerging at around $1210 \mathrm{~cm}^{-1}$ observed for the heavily boron-doped NCD films was previously reported for highly boron-doped single and polycrystalline diamond films. ${ }^{34-36}$ Some authors have attributed the $1200 \mathrm{~cm}^{-1}$ band to B-C complexes. ${ }^{36}$ B-C complexes were already reported in heavily boron-doped polycrystalline diamond films ${ }^{35}$ and have been more recently related to the origin $1210 \mathrm{~cm}^{-1}$ band in diamondlike B-C films obtained from the graphitelike B-C phase in a laser-heated diamond-anvil cell. ${ }^{43}$ From Figs. 2 and 3 it can be concluded that the intensity of the $G$ and $D$ bands is significantly lower in NCD than in UNCD, indicating lower $s p^{2}$ content in NCD films. This is not surprising, since the density of grain boundaries in UNCD is larger due to the smaller grain size. ${ }^{14,31}$ Grain boundaries were already suggested to have a strong influence on Raman characterization as well as in the electronic properties of UNCD films. ${ }^{31}$ As in the case of the first-order diamond line, both the $D$ and $G$ bands shift slightly toward lower wave numbers with the increase in the boron-doping level. For the $G$ band, this shift could suggest a slow increase in the bond disorder, ${ }^{33}$ which could be tentatively assigned to the presence of boron in the grain boundaries. Two peaks around 1150 and $1450 \mathrm{~cm}^{-1}$ are detected only in NCD and UNCD films and have been attributed to the presence of hydrocarbon layers. The visible Raman spectroscopy $(514.5 \mathrm{~nm}$, not shown in the graph) reveals both 1150 and $1450 \mathrm{~cm}^{-1}$ peaks, although they are slightly shifted to lower and to higher wave numbers, respectively. For the NCD film with the highest boron doping both peaks are hardly visible, which could be the result of a lower $\mathrm{H}$ incorporation in the grain boundaries.

Figure 4 presents examples of AFM images of the NCD films with different boron doping, from nonintentionally doped NCD film (S2) up to the heavily doped NCD film (S12) $\left([\mathrm{B}]_{\mathrm{SIMS}}=3.3 \times 10^{21} \mathrm{~cm}^{-3}\right)$. The surface roughness was estimated as a root mean square (rms) from the 1 $\times 1 \mu \mathrm{m}^{2}$ images. The rms values do not vary noticeably with the doping concentration and are in the range of 15-25 $\mathrm{nm}$. The grain size estimated from the AFM images yields a value between 130 and $160 \mathrm{~nm}$. No influence of the boron doping on the grain size was observed.

\section{B. Electronic properties}

\section{High-temperature regime}

The temperature-dependent conductivity of the NCD samples investigated in the high-temperature regime is presented in Fig. 5, which shows the influence of boron on the electronic properties of NCD. By increasing the boron concentration it is possible to vary the conductivity from 1.5 $\times 10^{-8} \Omega^{-1} \mathrm{~cm}^{-1}$ in the NID film (S2) up to $76 \Omega^{-1} \mathrm{~cm}^{-1}$ in the heavily doped NCD film (S12), as measured at room temperature. The temperature dependence of the conductivity of heavily boron-doped NCD films suggests the possibility of metalliclike behavior (described below in more detail) in agreement with the observation of the Fano resonance in the Raman spectra of the heavily doped NCD film. At high temperatures (300-700 K), the conductivity of the NID NCD film (S2) follows an exponential dependence with $1 / T$, with an activation energy very close to the expected value of the ionization energy for substitutional boron acceptors in single-crystalline or polycrystalline diamond, which is 370 meV. ${ }^{37,44}$ As mentioned before, SIMS revealed that the NID film has a boron concentration of $9.7 \times 10^{16} \mathrm{~cm}^{-3}$, resulting from the permanent contamination of the chemical vapor deposition (CVD) reactor with boron. However, for the 

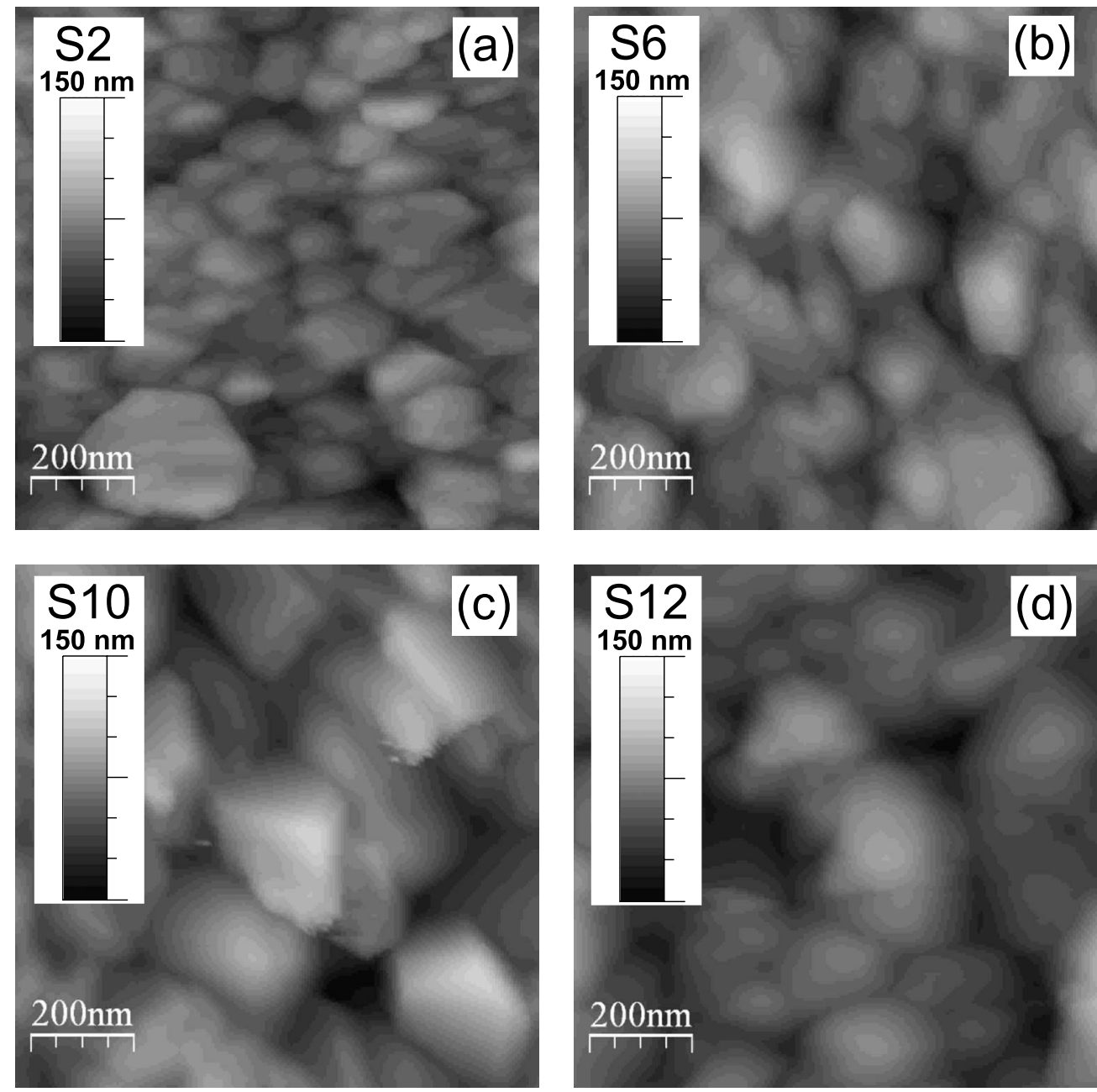

FIG. 4. AFM images of the (a) nonintentionally doped NCD film S2, (b) intermediate boron-doped NCD film S7 $\left(2.3 \times 10^{20} \mathrm{~cm}^{-3}\right),(\mathrm{c})$ heavily doped S10 $\left(1.3 \times 10^{21} \mathrm{~cm}^{-3}\right)$, (d) and heavily doped NCD film S12 ([B $\left.]_{\mathrm{SIMS}}=3.3 \times 10^{21} \mathrm{~cm}^{-3}\right)$. The measurements were performed at ambient conditions; $1 \times 1 \mu \mathrm{m}$ images were recorded.

boron-free NCD film (S1) an activation energy of about 0.7 $\mathrm{eV}$ was estimated at high temperatures, which is clearly larger than the ionization energy of substitutional boron (380 $\mathrm{meV}$ ) but lower than the $1-1.7 \mathrm{eV}$ reported for high quality intrinsic PCD films. ${ }^{22}$ Increasing the boron content in the gas phase results in a decrease in the apparent activation energy calculated in the high-temperature region (the values of the activation energies are listed in Table I). For the heavily doped film (S12), with a boron concentration of 3.3 $\times 10^{21} \mathrm{~cm}^{-3}$, the activation energy is about $12 \mathrm{meV}$. At low temperatures, however, the temperature dependence of the conductivity is almost vanished for the highest doped sample S12. At very low temperatures, the heavily boron-doped NCD films show superconductivity. The variation of the conductivity with the boron concentration is in good agreement with the data previously reported for single- and polycrystalline diamond films (see for instance the reviews by Nebel, ${ }^{22}$ Thonke, ${ }^{44}$ or Lagrange et al. ${ }^{19,20}$ When discussing the effect of the boron doping on the electronic properties of diamond, most of the authors refer to a model initially proposed by Visser et al. ${ }^{17}$ and successfully applied later on by Borst and Weiss. ${ }^{18}$ In this model, the conductivity can be described by the contribution of two main components: valence-band conduction and hopping transport. ${ }^{17}$

Motion of holes in extended states of the valence band represents the typical transport mechanism of a $p$-type semiconductor at high temperature. If a certain degree of compensation is present, the activation energy is determined by the ionization energy of the acceptor (for boron in diamond, $E_{A}=370 \mathrm{meV}$ ). This situation, however, is only valid for weakly boron-doped NCD films. For instance, the apparent activation energy of the temperature-dependent conductivity of the NID film (S2) is $E_{A}=390 \mathrm{meV}$. As the boron concentration increases, the activation energy calculated in the hightemperature range (from 295 up to $700 \mathrm{~K}$ ) decreases down to $12 \mathrm{meV}$ for heavily doped NCD films. The observed trend is consistent with the expected metal-insulator transition (Mott transition), which in the case of polycrystalline diamond films is expected for a boron concentration between $10^{20}$ and $10^{21} \mathrm{~cm}^{-3} \cdot 35$

The activation energy decrease with doping was originally discussed for silicon by Pearson and Bardeen, ${ }^{45}$ who proposed the following dependence of the effective activation energy on dopant concentration, $N_{A}$, 


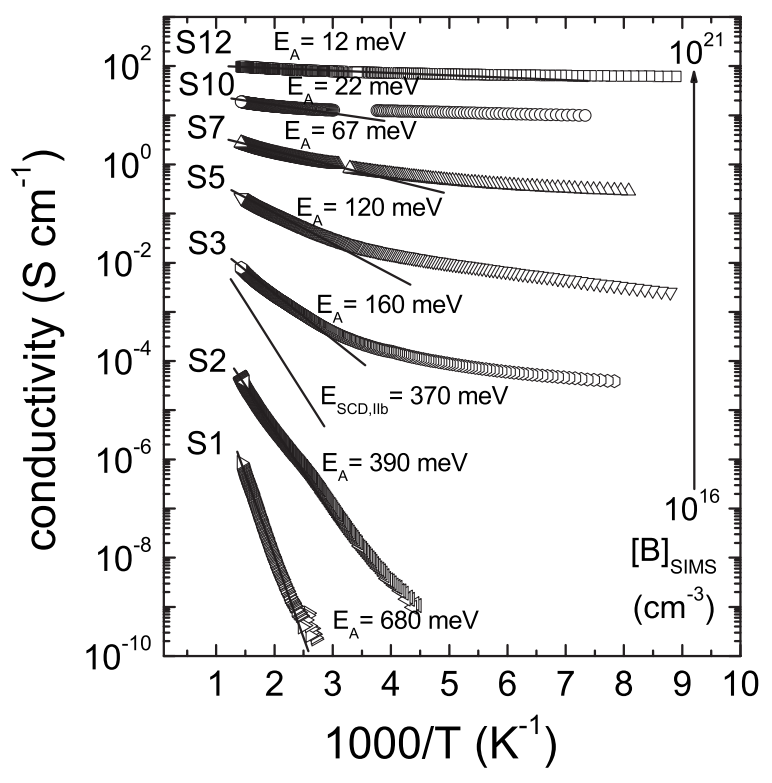

FIG. 5. Temperature dependence of the conductivity measured in NCD films. The boron concentration change derived by SIMS experiments is marked on the right side of the graph. The activation energies $E_{A}$ were calculated for the high-temperature range of the conductivity dependence.

$$
E_{A \text { eff }}=E_{I}-\alpha\left(N_{A}\right)^{1 / 3},
$$

where $E_{I}$ corresponds to the ionization energy for an isolated impurity center and $\alpha$ is a constant which was determined theoretically. ${ }^{45}$ This model was applied to diamond by Lagrange et al. ${ }^{19,20}$ and Thonke ${ }^{44}$ using $E_{I}=370 \mathrm{meV}$ for substitutional boron; for the case of diamond, the proportionality factor $\alpha$ calculated theoretically is $6.7 \times 10^{-8} \mathrm{eV} / \mathrm{cm}$. Although a qualitative agreement with the experimental data was obtained, this model was not able to fully explain the experiments. The discussion of Pearson and Bardeen ${ }^{45}$ was later on modified by Lee and McGill. ${ }^{46}$ It was shown that at least three other mechanisms should be considered to correctly account for the concentration dependence of the activation energy: the broadening of the impurity level, the tailing of the band edge, and the shift of the band edge. ${ }^{46}$ The broadening of the impurity level was demonstrated to be the most important term and is induced by the overlap between the impurity wave functions. This overlapping is proportional to the mean distance between the impurities and thus proportional to $\left(N_{A}\right)^{-1 / 3}$. Thus, the prediction of the Lee and $\mathrm{McGill}^{46}$ model closely resembles the dependence given in Eq. (1).

Therefore, and for the sake of simplicity, we use Eq. (1) to fit the activation energies shown in Fig. 6 as a function of the boron concentration. In the figure, the activation energies reported for single crystalline diamond films ${ }^{18-20}$ and polycrystalline diamond ${ }^{47,48}$ are compared with the experimental data observed in this study for NCD films. The prediction of Eq. (1) using the theoretically estimated value of $\alpha, 6.7$ $\times 10^{-8} \mathrm{eV} / \mathrm{cm}$, represented in Fig. 6 by the dashed line, underestimates the values of the measured activation energies for the heavily boron-doped NCD. Using Eq. (1) we have

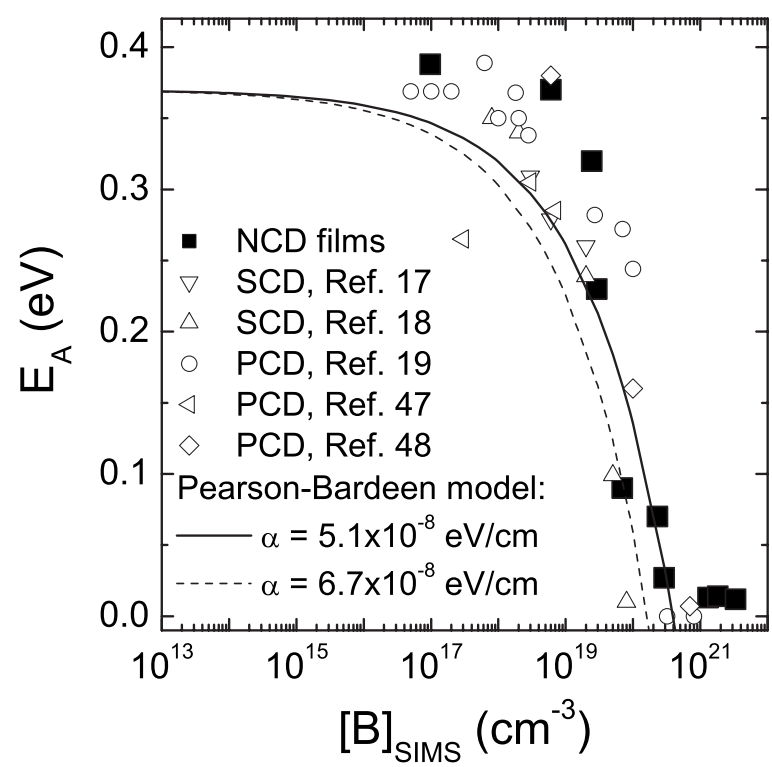

FIG. 6. Dependence of the conductivity activation energy, $E_{A}$, on the boron doping in NCD films. The activation energies were calculated for the high-temperature range of the conductivity dependence (see Fig. 4). A theoretical curve according to Eq. (3) is given by the solid curve. Experimental data reported for homoepitaxial diamond films (Refs. 17-19) and polycrystalline diamond films (Refs. 47 and 48) are plotted for comparison.

performed a best fit to the data resulting in a value of $\alpha$ $=5.1 \times 10^{-8} \mathrm{eV} / \mathrm{cm}$ (solid line Fig. 6). The predicted metalinsulator transition $\left(E_{A}=0\right)$ occurs for doping concentrations of about $4 \times 10^{20} \mathrm{~cm}^{-3}$, in good agreement with the conductivity and carrier-concentration data that will be discussed later. Figure 6 suggests that the Pearson-Bardeen model provides a reasonable explanation for the decrease in the activation energy in nanocrystalline diamond films even if it underestimates the activation energy at low boron concentrations. It is important to note that in the case of the heavily doped NCD films (above the Mott transition), the experimental activation energy is significantly higher than predicted by Eq. (1) and observed for single-crystal and polycrystalline films. The reason for the temperature activated conductivity of the heavily doped NCD observed at high temperatures is so far not clear, but it could result from the contribution of the potential barriers introduced by the grain boundaries, as previously discussed for polycrystalline diamond films. ${ }^{22}$

The second contribution to the conductivity is due to hopping transport between boron acceptor states and is expected to be the dominant transport mechanism at low temperature for samples with a boron concentration slightly below the Mott transition. Since the hopping process occurs between occupied and unoccupied acceptor states, it is strongly dependent on the compensation ratio of the diamond films. Hopping transport is usually described by nearest-neighbor hopping $(\mathrm{NNH})$ and variable range hopping (VRH). Both are expected to be strongly dependent on the distance between the acceptor atoms. ${ }^{18,49}$ In the case of diamond, the boron acceptor activation energy is relatively high, enabling the $\mathrm{NNH}$ mechanism to be observed even at room temperature. 

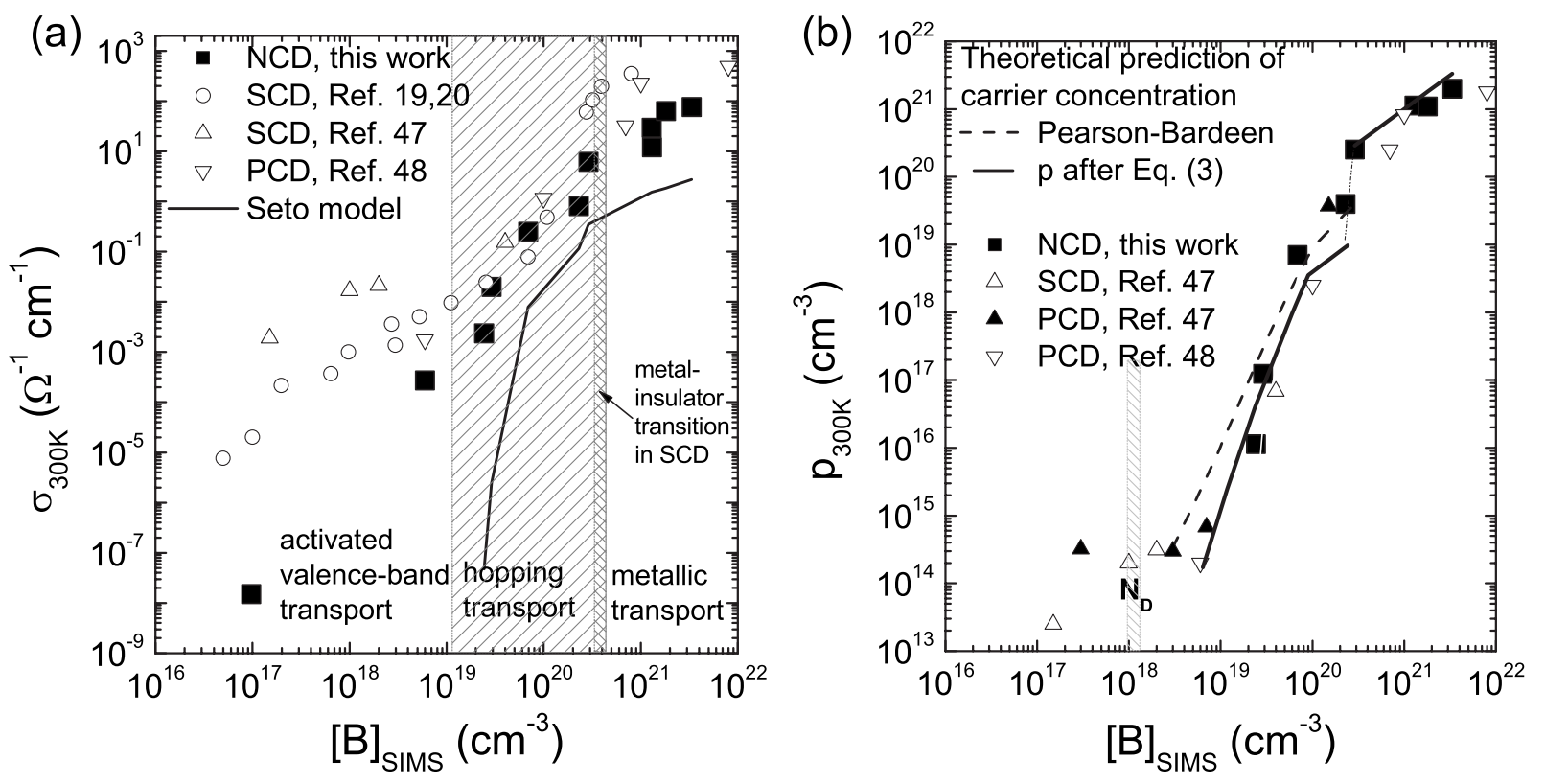

FIG. 7. (a) Room-temperature conductivity and (b) carrier concentration as a function of boron doping measured for NCD films. Values reported in the literature are plotted for comparison (Refs. 19, 20, 47, and 48). The position of the onsets for the changes from valence-band conduction to hopping conduction as well as from hopping conduction into metallic conduction as predicted by theoretical model (Refs. 20 and 43) are marked with the dashed lines. The curve predicted by the Seto model of conductivity [see Eq. (4) and Ref. 40] is plotted in (a) as a solid line. A solid line in (b) represents the expected carrier concentration calculated using Eq. (3) using the activation energy obtained from temperature dependence of carrier concentration and a dashed line the carrier concentration calculated within the Pearson-Bardeen model (Ref. 45).

The VRH process was observed in different diamond films, typically at temperatures lower than $150 \mathrm{~K}^{45}$

We will discuss now a third contribution to the conductivity in NCD, which accounts for the hopping conduction in the grain boundaries previously identified in undoped UNCD films as the main conduction path. ${ }^{15,31}$ In high quality PCD films, where neither nitrogen nor boron dopants contribute to conduction, the measured activation energy of the conductivity, $E_{A} \sim 1-1.7 \mathrm{eV}$, was attributed to the thermal activation of carriers in $\pi-\pi^{*}$ bands introduced by $s p^{2}$-bonded carbon present in the grain boundaries. ${ }^{22}$ The room-temperature conductivities of single crystalline diamond films, ${ }^{19,47}$ polycrystalline diamond films, ${ }^{48}$ and our NCD films are shown as a function of boron concentration in Fig. 7(a). The range of boron concentrations where different conduction mechanisms are expected to dominate are marked in Fig. 7(a), taking into account the most recently reported values for the metal-insulator transition (marked as mit) in single-crystal diamond films, between $2 \times 10^{20}$ and $4.5 \times 10^{20} \mathrm{~cm}^{-3} \cdot 23,24$ As the graph reveals, the conductivity of the weakly borondoped NCD films is about 3 orders of magnitude lower compared to homoepitaxial boron-doped films. For boron concentrations where hopping transport is expected to be dominant [shadowed region in Fig. 7(a)], the conductivity of NCD is very close to the conductivity of polycrystalline and single crystalline diamonds. For the heavily doped NCD films, already in the metalliclike transport regime, the conductivity of the NCD films is about 1 order of magnitude lower.

The dependence of the hole carrier density (as measured by the Hall-effect experiments) on the boron concentration for NCD, SCD, and PCD films is plotted in Fig. 7(b). Due to the limitations imposed by the very high resistivity of low doped NCD films, Hall-effect experiments were only possible for samples with $\mathrm{B}: \mathrm{C}$ atomic density higher than 2.5 $\times 10^{19} \mathrm{~B} / \mathrm{cm}^{3}$. At room temperature, the resulting carrier concentration ranges from $7 \times 10^{15} \mathrm{~cm}^{-3}$ for a weakly doped (S4) film up to $2 \times 10^{21} \mathrm{~cm}^{-3}$ in the heavily doped (S12) NCD film. It can be observed that the carrier concentration measured in NCD films is comparable to the values measured in SCD and PCD. The large scattering of values observed in Fig. 7(b) can be explained by the uncertainty of the SIMS concentration values as well as of the carrier concentration calculated from the Hall experiments. The value of the boron concentration at which the conductivity mechanism changes from hopping conduction to metal-like conduction is around $4 \times 10^{20} \mathrm{~cm}^{-3}$, as estimated from Figs. 6 and 7 , which is in good agreement with the values reported for boron-doped SCD diamond films. ${ }^{19,20,23,24}$

To calculate the expected concentration of holes in NCD, the condition of neutrality for a $p$-type diamond with compensation was used together with expression for the hole concentration in a nondegenerate $p$-type semiconductor, resulting in

$$
\frac{p\left(p+N_{D}\right)}{N_{A}-N_{D}-p}=\left(\frac{2 \pi m_{h}^{*} k_{B} T}{h^{2}}\right)^{3 / 2} \exp \left(-\frac{E_{A}}{k_{B} T}\right) \equiv N_{x}
$$

which yields an expression for the hole density $p$, 

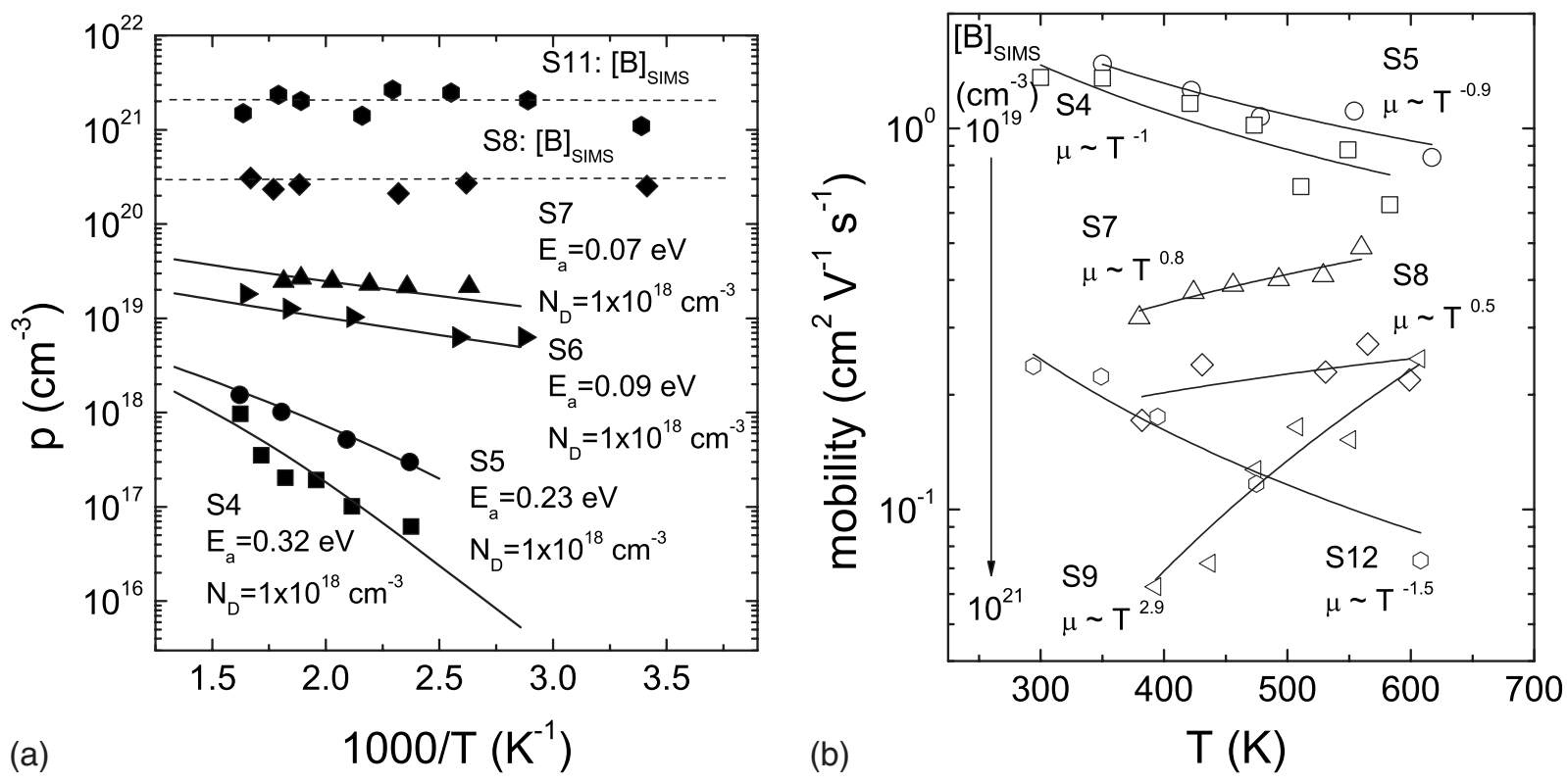

FIG. 8. Temperature dependence of the (a) carrier (hole) concentration and (b) carrier mobility measured in temperature range from 295 to $650 \mathrm{~K}$. The solid lines in (a) represent best fit according to Eq. (3). The fitting parameters are summarized in the graph. For heavily boron-doped NCD films boron concentrations derived from SIMS experiments are marked by dashed lines. The lines in (b) correspond to best fits of the temperature-dependent mobility using $\mu \sim T^{\gamma}$, where the $y$ parameters are given in the graph for each NCD film.

$$
p=\frac{N_{D}+N_{x}}{2}\left(\sqrt{1+\frac{4 N_{x}\left(N_{A}-N_{D}\right)}{\left(N_{D}+N_{X}\right)^{2}}}-1\right),
$$

where $N_{A}$ is the boron doping, $N_{D}$ the compensating donor density, $E_{A}$ the activation energy of the hole concentration, and $m_{h}^{*}$ the hole effective mass. A concentration of compensating donors of $N_{D}=1 \times 10^{18} \mathrm{~cm}^{-3}$ and $m_{h}^{*}=0.7 m_{e}$ were used for calculations. The carrier concentrations for NCD films with low and intermediate dopings calculated according to Eq. (3) are plotted in Fig. 7(b) as a solid line. For the calculations, we used the activation energy obtained from the fit of the temperature dependence of the carrier concentration measured with Hall effect, as shown in Fig. 8(a) and discussed below. As can be seen in Fig. 7(b), within experimental accuracy, the data are in good agreement with the calculated values corresponding to the lower doping level (films $\mathrm{S} 4$ and S5) and the intermediate boron-doped NCD films (S6). In the calculations a constant value of the compensating donors was assumed, which is not necessarily the case: it is possible that for weakly doped NCD films the concentration of the donors, $N_{D}$, is higher than assumed. For the heavily doped films (S7-S12) above the metal-insulator transition, however, an underestimation of the calculated carrier density is expected as a result of the assumption of a nondegenerate system used to derive Eqs. (2) and (3). Thus, for boron concentrations above the metal-insulator transition, it can be assumed that the number of free carriers corresponds to the number of impurities if compensation is negligible. In Fig. 7(b), the solid line shown for boron concentrations above $3 \times 10^{20} \mathrm{~cm}^{-3}$ corresponding to the calculated value of the density of holes assuming $p=N_{A}$ is in very good agreement with the experimentally determined carrier concentration. The dashed line in Fig. 7(b) was calculated according to
Eq. (3) using the $E_{A}$ values of the Pearson-Bardeen model obtained from Eq. (1) with $\alpha=5.1 \times 10^{-8} \mathrm{eV} / \mathrm{cm}$. The calculated values are also in good agreement with the experimental data for boron-doped NCD films below the metalinsulator transition, suggesting that the Pearson-Bardeen model can be used to predict the increase in the carrier concentration with boron doping, which results from the lowering of the activation energy due to the decrease in the average distance between the acceptors.

The temperature dependence of the carrier concentration and mobility calculated from Hall-effect experiments are shown in Figs. 8(a) and 8(b) for temperatures between 295 and $700 \mathrm{~K}$. In general, heavily doped samples show a weak temperature dependence of the carrier concentration, in good agreement with the results of the conductivity. Equation (3) was used to fit the experimental data of low and intermediate boron-doped NCD films (S4-S7). The fitting parameters are shown in the graph for each NCD film. For the low doped NCD films S4 and S5, more pronounced temperature dependence is observed in the high-temperature regime and the activation energy of the carrier concentration gets closer to $370 \mathrm{meV}$, as expected for a compensated boron-doped diamond. As discussed before, for NCD films with boron concentration above the Mott transition (S8-S12) we assume that $p \approx N_{A}$. The experimentally determined carrier concentration for the heavily boron-doped NCD films S8 and S11 is also plotted in Fig. 8(a) together with the boron concentration $[\mathrm{B}]_{\text {SIMS }}$ determined by SIMS (dashed lines). A good agreement between the Hall-effect data and SIMS is observed, confirming the expected metal-like behavior of the electronic transport in heavily doped NCD films.

On the other hand, it is important to determine whether the $s p^{2}$ amorphous carbon phase of the grain boundaries contributes or not to the conduction process of the heavily doped 
sample, as is the case for ultrananocrystalline diamond films. ${ }^{31}$ In UNCD films the conductivity has been attributed to the presence of $s p^{2}$ carbon in the grain boundaries which increases with the amount of nitrogen in the gas phase. ${ }^{15,16}$ Thus, it has been shown that electron transport occurs in a defect band at the grain boundaries. In contrast, our Halleffect experiments confirm that in the B-doped NCD films the conductivity is due to holes and not electrons, suggesting that electronic transport in the grain boundaries is not an important transport mechanism in B-doped NCD. In addition, from the analysis of the Raman data (Fig. 2) we have concluded that the amorphous carbon content does not increase significantly with the boron concentration and therefore its contribution to the conductivity of heavily doped NCD can be neglected. Figure 8(b) shows the temperature dependence of the hole mobility, as calculated from the Halleffect experiments. The mobility values are roughly 1-2 orders of magnitude lower than the mobility of single-crystal diamond films with comparable doping levels, so that additional scattering mechanisms dominate over the basic mechanisms such as impurity scattering and phonon scattering. The lines in Fig. 8(b) correspond to best fits of the temperaturedependent mobility using $\mu \propto T^{\gamma}$. The values of the exponent $\gamma$ are shown in Fig. 8(b). The expected temperature dependence of the mobility for acoustic phonon scattering is $\mu$ $\propto T^{-3 / 2}$ and for ionized impurities scattering $\mu \propto T^{3 / 2}{ }^{50}$ From Fig. 8(b), it is evident that the interpretation of the temperature-dependent mobility data is rather difficult and a reliable conclusion on the dominant scattering mechanism is not possible.

In order to verify the possible influence of the grainboundary scattering and ionized acceptor scattering on the free-carrier mobility the experimental data at room temperature have been plotted in Fig. 9 as a function of boron concentration, together with the theoretical predictions of ionized impurity scattering and grain-boundary scattering expected for polycrystalline material. ${ }^{40}$ The carrier mobility dependence on boron doping for single crystalline ${ }^{47}$ and polycrystalline ${ }^{48}$ diamond films have also been plotted in Fig. 9 for comparison. The hole mobility decreases with doping, from $2 \mathrm{~cm}^{2} \mathrm{~V}^{-1} \mathrm{~s}^{-1}$ in a low boron-doped sample (S4) to $0.2 \mathrm{~cm}^{2} \mathrm{~V}^{-1} \mathrm{~s}^{-1}$ in the highest doped sample (S12). The observed decrease in the carrier mobility with increasing boron concentration is qualitatively consistent with the behavior of single- and polycrystalline diamond films. ${ }^{18,20,22}$ However, due to the nanocrystalline structure of NCD films, the scattering at the grain boundaries is expected to be the main cause of the relatively low hole mobility measured for the low B-doped NCD films. For comparison, in weakly doped polycrystalline diamond films (hole density in the range of $4 \times 10^{15} \mathrm{~cm}^{-3}$ ), the hole mobility is close to $40 \mathrm{~cm}^{2} \mathrm{~V}^{-1} \mathrm{~s}^{-1} \cdot 22$ Compared to single crystalline diamond with the same boron concentration, the mobility in NCD is of 3 orders of magnitude lower. For the NCD film S4 with hole density in the range of $10^{15} \mathrm{~cm}^{-3}$ the mobility is about $2 \mathrm{~cm}^{2} \mathrm{~V}^{-1} \mathrm{~s}^{-1}$. For increasing boron concentration, however, the mobilities of SCD, PCD, and NCD get closer. We have considered the influence of the grain boundaries within the Seto model, which was originally introduced to explain the electronic transport of B-doped micro- and nanocrystalline

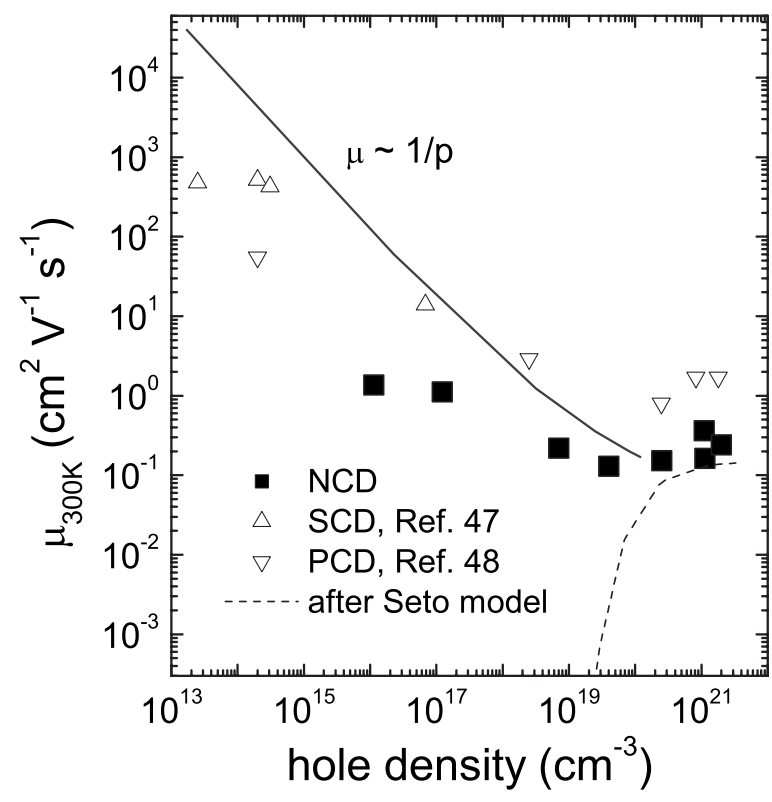

FIG. 9. Hole mobility dependence on hole concentration in NCD films. The data for B-doped single-crystal (Ref. 47) and polycrystalline diamond films (Ref. 48) available in the literature were plotted for comparison. The dashed line represents the expected mobility dependence $\mu \sim 1 / p$ due to ionized acceptors. Dashed line represents the mobility [Eq. (4)] curve predicted by the Seto model.

silicon. ${ }^{40}$ Charge trapping at grain boundaries, which leads to the formation of potential barriers, is responsible for the measured high activation energies (much higher than expected for substitutional boron acceptors in $\mathrm{Si}$ ) in polycrystalline silicon films. ${ }^{40}$ For a given crystallite size $(L)$, a critical value of boron doping $N_{A c}$ must be exceeded to prevent the crystal regions from total depletion of carriers. This results in an abrupt increase in free-carrier density and conductivity of the polycrystalline silicon films at $N_{A c}$. The carrier mobility estimated by Seto is given by ${ }^{40}$

$$
\mu_{a}=L q\left(\frac{1}{2 \pi m_{h}^{*} k_{B} T}\right)^{1 / 2} \exp \left[\frac{-q Q^{2}}{8 \varepsilon k_{B} N_{A} T}\right],
$$

where $Q$ corresponds to the density of traps in the grain boundaries. Using $L=150 \mathrm{~nm}$ and $Q=5 \times 10^{13} \mathrm{~cm}^{-2}$, and a hole effective mass $m_{h}^{*}=0.7 m_{e},{ }^{18}$ the prediction of Eq. (4) is plotted in Fig. 9 as a dashed line. Should the mobility be exclusively dependent on the potential barriers introduced by the grain boundaries, as modeled by Seto, a minimum of the mobility would be expected at $N_{A c}{ }^{40}$ From the experimental data of the B-doped NCD films no such a mobility minimum is observed. On the other hand, the decrease in the carrier mobility observed for NCD films stays in reasonable agreement with the expected mobility lowering as a result of the ionized acceptors scattering. The solid line in Fig. 9 corresponds to the mobility calculated assuming ionized impurity scattering using ${ }^{50}$ 


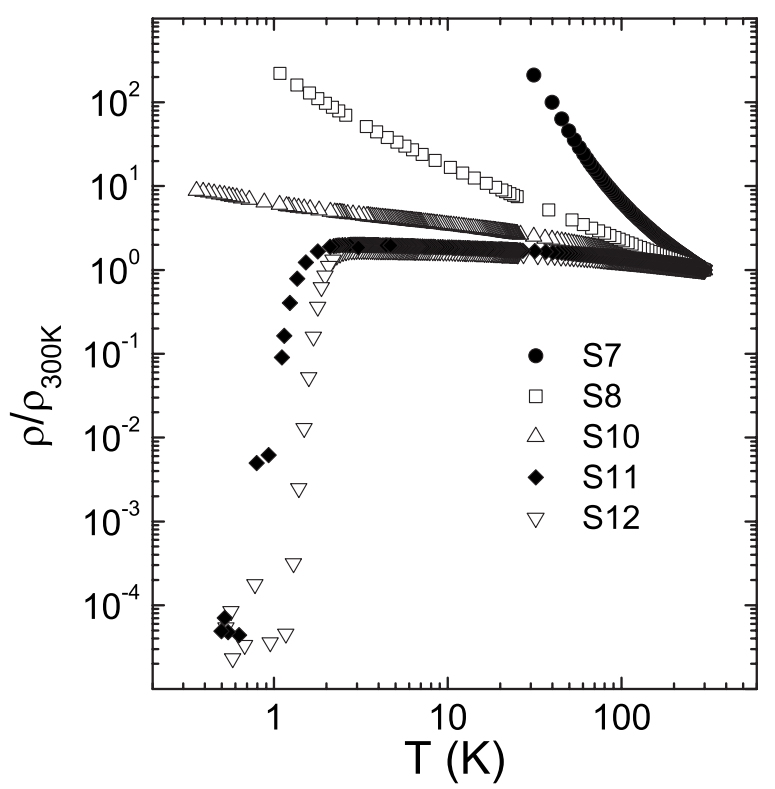

FIG. 10. Temperature dependence of the normalized resistance $\rho / \rho(300 \mathrm{~K})$ from $300 \mathrm{~K}$ down to $50 \mathrm{mK}$. Highly doped samples with doping concentration above $10^{21} \mathrm{~cm}^{-3}$ show superconductivity with a critical temperature of about $2 \mathrm{~K}$.

$$
\mu_{\text {ionized }}=\frac{64 \sqrt{\pi} \varepsilon_{0}^{2}(2 k T)^{3 / 2}}{p q^{3} m_{h}^{* 1 / 2}}\left\{\ln \left[1+\left(\frac{12 \pi \varepsilon_{0} k T}{q^{2} p^{1 / 3}}\right)^{2}\right]\right\}^{-1},
$$

in which a density of ionized impurities, $p$, calculated using Eq. (3), $\varepsilon_{0}=5.7$, and an effective hole mass of $m_{h}^{*}=0.7 m_{e}$ were used. It can be seen that the decrease in the mobility in SCD and PCD films (Ref. 22 and references therein) also follow the trend predicted by Eq. (5).

In addition, the conductivity calculated using the Seto model [dashed line in Fig. 7(a)] predicts a sharp decrease in conductivity which does not correspond to the experimental data. The disagreement of the carrier mobility and conductivity values calculated using the Seto model with the experimental data can have several reasons: (i) too large size of the diamond crystallites $(L>100 \mathrm{~nm})$ for which the Seto model fails ${ }^{47}$ and (ii) the Seto assumption of a full acceptors ionization $p=N_{A}$, which in the case of boron-doped NCD films with a relatively high acceptor ionization energy and weak compensation is not valid. Alternatively, a modified Seto model could be used (Ref. 47 and references therein) taking into account carrier scattering processes depending on the ratio between the grain size and the carrier depletion region size. From our experimental data it is not possible to perform a more detailed study of the influence of the grain-boundary trapping on the electronic properties of B-doped NCD films. It would be necessary to perform a comprehensive study of the electronic properties of NCD films with low and intermediate boron dopings as well as with smaller grain size in order to investigate a possible influence of the charge trapping effect induced by the grain boundaries. Currently, we cannot rule out the effect of charge trapping at grain boundaries at low boron-doping levels below $10^{18} \mathrm{~cm}^{-3}$.

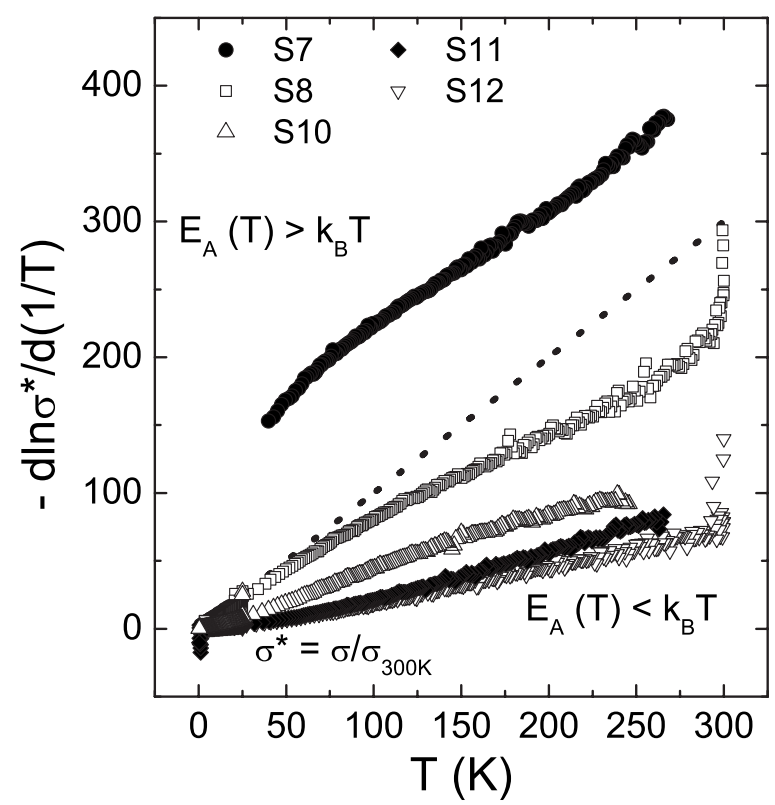

FIG. 11. Effective activation energy $E_{\text {act }}$ as determined from the slope of the Arrhenius plot of the temperature-dependent conductivity normalized to its room-temperature value. Only weakly doped samples (S7) show thermally activated hopping conduction, whereas higher doped samples behave like disordered metals.

\section{Low-temperature regime}

The temperature dependence of the normalized resistance $\rho / \rho(300 \mathrm{~K})$ in the range $300 \mathrm{~K}$ down to $350 \mathrm{mK}$ is shown in Fig. 10 for several samples. In contrast to the minimum resistivity observed in single-crystal diamond at intermediate temperatures, ${ }^{24}$ the resistivity of nanocrystalline diamond increases with decreasing temperature. The highly doped samples with $n_{B} \geq 1.3 \times 10^{21} \mathrm{~cm}^{-3}$ also show superconductivity with a critical temperatures of about $T_{c}=2.1 \mathrm{~K}$ (samples S8 and S10 behaved like a disordered metal, but showed no superconductivity down to $350 \mathrm{mK}$ ). Figure 11 shows the effective activation energy $E_{\text {act }}$ as determined from the slope of Arrhenius plots of the temperature-dependent conductivity $\sigma^{*}$ normalized to its room-temperature value. As mentioned in Sec. III B 1, thermally activated transport via hopping conduction $\left(E_{\text {act }}>k_{B} T\right)$ is only observed for the weakly doped sample S7. In contrast to the classical behavior of an insulator, the apparent activation energy of a metal is only a small fraction of $k_{B} T$ in the corresponding temperature range, as already reported elsewhere. ${ }^{51-53}$ The highly doped samples behave like a disordered metal $\left(E_{\text {act }}<k_{B} T\right)$. Therefore, the critical boron concentration $n_{c}$ for the metalinsulator transition, as obtained from the low-temperature experiments, lies in the range from $2.3 \times 10^{20}$ up to 2.9 $\times 10^{20} \mathrm{~cm}^{-3}$, in good agreement with the results discussed in previous sections, and with what was found for single-crystal and polycrystalline diamonds (Refs. 54 and 55). Due to the granularity of the diamond thin film, we expect that the metal-insulator transition is not only due to the increasing doping within the grains, but also to the increase in intergranular coupling between metallic grains. 


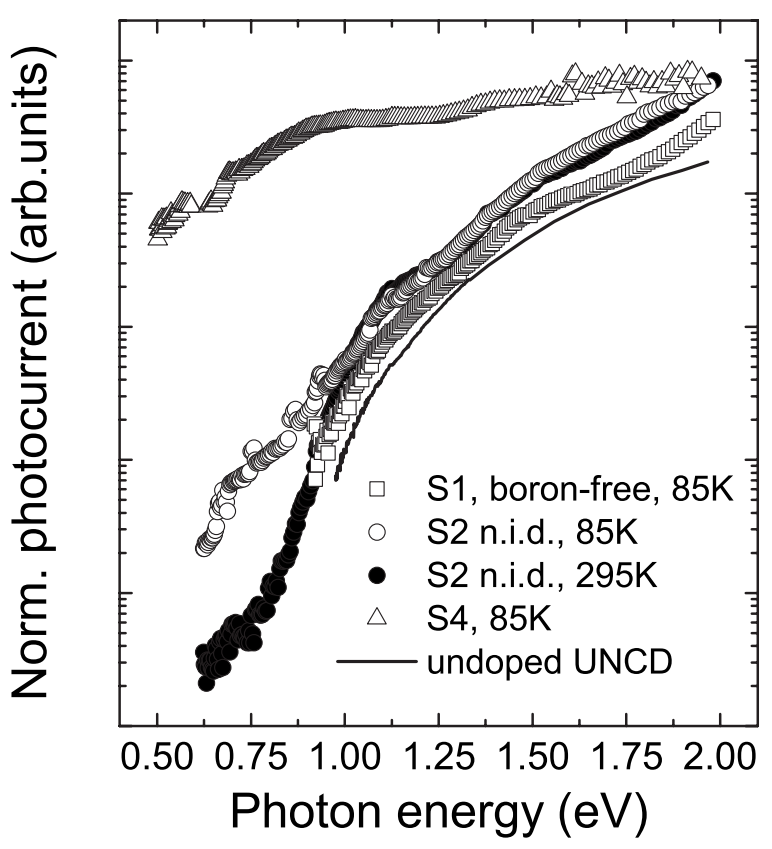

FIG. 12. Normalized spectrally resolved photocurrent in "asgrown" NCD films with different boron concentration (boron-free, nonintentionally doped-9.7 $\times 10^{16} \mathrm{~cm}^{-3}$ and low boron doped $-2.7 \times 10^{19} \mathrm{~cm}^{-3}$ ) measured at $85 \mathrm{~K}$. The spectrum of nonintentionally doped NCD film S2 at $295 \mathrm{~K}$ was plotted to show a temperature dependence of the photocurrent signal observed as an onset at about $0.6 \mathrm{eV}$. The spectrum of the UNCD film is presented to compare the influence of the amorphous carbon in the grain boundaries on the photocurrent signal. The spectra were shifted vertically to emphasize the differences.

\section{Optical properties}

Spectrally resolved photocurrent experiments were performed using boron-free nonintentionally doped (NID) $\left([\mathrm{B}]_{\text {SIMS }}=9.7 \times 10^{16} \mathrm{~cm}^{-3}\right)$ and low boron-doped $\left([\mathrm{B}]_{\text {SIMS }}\right.$ $<2.7 \times 10^{19} \mathrm{~cm}^{-3}$ ) NCD films. The spectra were obtained from 0.35 to $6 \mathrm{eV}$ for temperatures between 80 and $360 \mathrm{~K}$. Photocurrent (PC) was not observed for heavily boron-doped samples, which can be due to the large background current and the short lifetime of photogenerated carriers. Figure 10 presents the photocurrent spectra of the boron-free film S1, the NID film S2, and the weakly boron-doped S4 NCD film. The spectra of the S1 and S2 NCD films show an onset at about $1.0 \mathrm{eV}$, which is comparable to the photocurrent signal measured in undoped ultrananocrystalline diamond films (black solid line in Fig. 12), ${ }^{31}$ and also similar to polycrystalline CVD diamond films. ${ }^{22}$ The onset at $1.0 \mathrm{eV}$ is tentatively assigned to the contribution of $s p^{2}$ bonded carbon in the grain boundaries and, thus, to transitions between the $s p^{2}$ related $\pi$ and $\pi^{*}$ states. In the case of the NID (S2) and low boron-doped (S4) NCD films, a clear photocurrent signal below $1.0 \mathrm{eV}$ is observed, with an intensity increasing as the boron concentration increases (Fig. 12). According to the work on polycrystalline diamond $\mathrm{d}^{29,30}$ and recent publications on Fourier transform photocurrent spectroscopy (FTPS) in nanocrystalline diamond,$^{56}$ the photocurrent signal at energies below $0.5 \mathrm{eV}$ is related to the presence of boron impu-

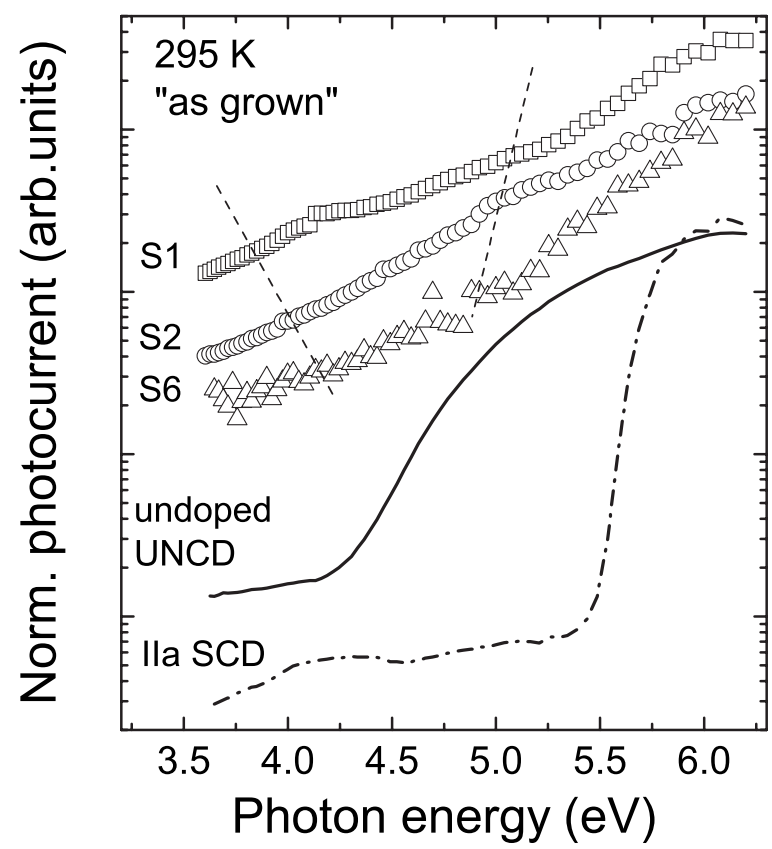

FIG. 13. Normalized spectrally resolved photocurrent in "asgrown" NCD films with different boron concentrations (boron-free, nonintentionally doped-9.7 $\times 10^{16} \mathrm{~cm}^{-3}$ and low boron doped-6.9 $\times 10^{19} \mathrm{~cm}^{-3}$ ) in the high energy range. A difference in the expected band-to-band absorption onset is present depending on the boron incorporation in the film. Shift to the lower energy is expected to originate from the absorption in the band tails due to disorder.

rities in the NCD films. The photocurrent in the range 0.5$0.9 \mathrm{eV}$ was previously referred by various authors to the contribution of a defect state $D_{x}{ }^{30,31,56}$ The photocurrent spectra of the S2 NCD film show the same temperature trend reported for the $D_{x}$ defect in NCD: an increase in the photocurrent in the range $0.6-0.9 \mathrm{eV}$ for decreasing temperatures (see Fig. 12). The observed increase in the photocurrent at lower temperatures is consistent with the model proposed by Kravets et al. ${ }^{29}$ which supported a rather broad distribution of gap states instead of a sharp defect level. Therefore, hole transitions to the valence-band shift to lower photon energy at lower temperatures since the Fermi level and quasi-Fermi level for holes move toward the valence-band edge with decreasing temperature. ${ }^{29}$ However, the origin of this $D_{x}$ defect is not understood and, thus, further investigation is required.

In the high energy range of the spectra two onsets can be observed (Fig. 13) at about 4.0 and $5.3 \mathrm{eV}$. The one at higher energy can be attributed to the indirect band to band transition of diamond. For NCD films with higher boron-doping concentration this transition slightly shifts to lower energies: for instance, the onset occurs at about $5.0 \mathrm{eV}$ for sample $\mathrm{S6}$, with $[\mathrm{B}]_{\mathrm{SIMS}}=6.9 \times 10^{19} \mathrm{~cm}^{-3}$. As shown in Fig. 13, the band to band transition for NCD films is not very pronounced, which is the result of the high photocurrent at lower energies as discussed before. As it has been suggested for polycrystalline $e^{22,57}$ and ultrananocrystalline ${ }^{31}$ diamond films, the nondiamond phase in the grain boundaries has a prominent influence on the optical absorption in diamond. For instance, it has been shown that the grain boundaries 


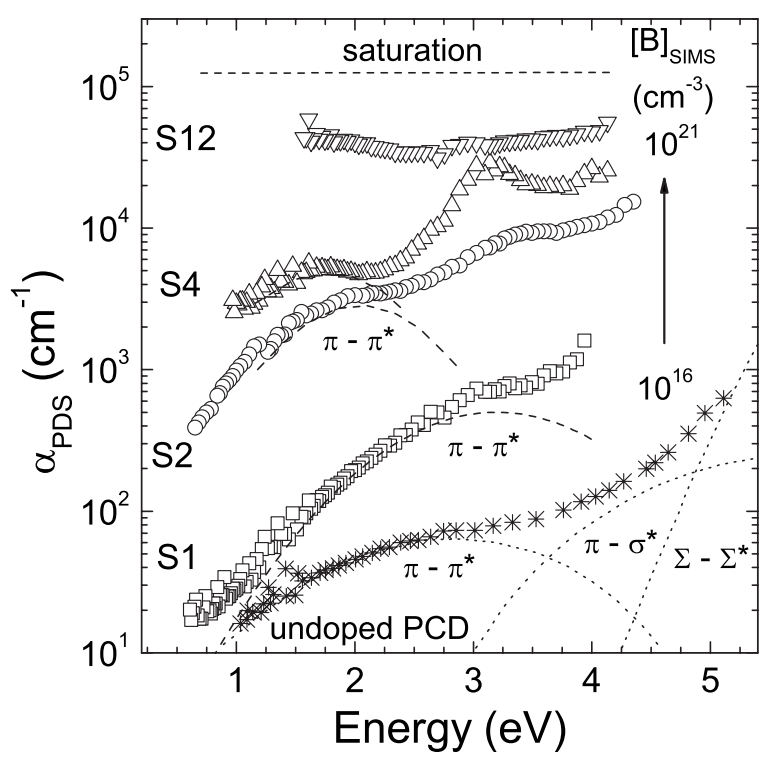

FIG. 14. PDS absorption coefficient $\alpha_{\text {opt }}$ calculated for NCD samples. The onset at about $0.8 \mathrm{eV}$ is attributed to transitions from the $\pi$ to $\pi^{*}$ band.

play a major role in the photocurrent of UNCD films. ${ }^{31}$ The PC spectrum of an undoped UNCD film is shown together with the NCD spectra in Fig. 13. In the UNCD film, the onset at about $4.1 \mathrm{eV}$ was assigned to transitions between valence and conduction-band tails, which are induced by the disordered $s p^{3}$ phase. ${ }^{31}$ For comparison, the photocurrent spectrum of a single crystalline diamond film (Fig. 13), in which the density of grain boundaries is much lower, shows a sharp band-to-band absorption onset at about $5.4 \mathrm{eV}$.

In order to determine the optical-absorption coefficient $\alpha_{\text {opt }}$ of boron-doped NCD films, PDS measurements were performed with the boron-free (S1), nonintentionally doped (S2, $\left.[\mathrm{B}]_{\mathrm{SIMS}}: 9.7 \times 10^{16} \mathrm{~cm}^{-3}\right)$, weakly boron-doped (S5, $[\mathrm{B}]_{\mathrm{SIMS}}<2.7 \times 10^{19} \mathrm{~cm}^{-3}$ ), and heavily boron-doped (S12, $[\mathrm{B}]_{\text {SIMS }}: 3.34 \times 10^{21} \mathrm{~cm}^{-3}$ ) NCD films. The calculated absorption coefficient is plotted in Fig. 14. We assume a similar energy dependence of the density of states (DOS) than that used for polycrystalline diamond films ${ }^{22,57}$ and undoped and nitrogen doped NCD and UNCD. ${ }^{31}$ Thus, bonding and antibonding, $\pi$ and $\pi^{*}, s p^{2}$-related states as well as dangling bonds introduced by the grain boundaries, all with a Gaussian shaped distribution, ${ }^{22,57}$ are expected to bring an important contribution to the total optical absorption of the nanocrystalline diamond films. As already discussed for the photocurrent spectra, the onset at about $1.0 \mathrm{eV}$ is attributed to transitions from $\pi$ to $\pi^{*}$ states. At higher energies, however, several optical transitions could be responsible for the optical absorption in the NCD films. In the case of nanocrystalline diamond, $s p^{2}$ and $s p^{3}$ sites occur together in the highly disordered grain-boundary regions and a sufficient bond angle distortion can enhance $\pi-\sigma$ bond mixing. This allows transitions from the $s p^{2}$-related $\pi$ states to the antibonding distorted $s p^{3}$ states or to the valence-band tail region. Also band tail to band tail and band-to-band transitions can contribute to the optical absorption at high energies. In the case of the intrinsic and NID NCD films, where $\pi$ and $\pi^{*}$
TABLE II. Fitting parameters of the boron-doped NCD films absorption (Fig. 14).

\begin{tabular}{lcl}
\hline \hline Sample & $E_{\Delta \pi}(\mathrm{eV})$ & $\omega(\mathrm{eV})$ \\
\hline S1 & 3.2 & 0.55 \\
S2 & 2.4 & 0.45 \\
S3 & 2.3 & 0.4 \\
S5 & 2.0 & 0.45 \\
\hline \hline
\end{tabular}

states are approximated by Gaussian distributions placed symmetrically around the Fermi level, ${ }^{22,31,57}$ the opticalabsorption coefficient for the transitions between $\pi$ and $\pi^{*}$ states was calculated using a joint density of states, ${ }^{22,31}$ with a characteristic pseudogap, $E_{\Delta \pi}=E_{\pi^{*}-E_{\pi}}$, between the $\pi$ and $\pi^{*}$ states $^{31}$ and the full width half maximum of the density distribution $\omega$. The results summarized in Table II are in good agreement with the photocurrent data as well as with previously reported data. ${ }^{22,31,57}$ The spectra of the boron-free NCD film S1 is dominated by a broad $\pi-\pi^{*}$ transition (Fig. 13), also observed in undoped PCD films, ${ }^{23}$ shown for comparison in Fig. 14. The value of the pseudogap estimated for $\mathrm{S} 1 \mathrm{NCD}$ film, $E_{\Delta \pi}=3.2 \mathrm{eV}$, is in the range of values calculated for undoped PCD, namely, 3.4-3.8 eV, suggesting a negligible influence of the amorphous carbon on the band structure in the case of intrinsic PCD and NCD films. In the case of the NID NCD film (S2) the contribution of $\pi-\pi^{*}$ transitions is clearly visible in the PDS spectra. The fitting of the $\pi-\pi^{*}$ transition yields $E_{\Delta \pi}=2.4 \mathrm{eV}$. The contribution of $\pi-\pi^{*}$ and $\pi-\sigma^{*}$ transitions was also observed in the PDS spectra of the intermediate boron-doped (S3 and S5) NCD films (not shown in the graph). As expected, the opticalabsorption coefficient $\alpha_{\text {opt }}$ increases with increasing boron concentration. For the heavily doped NCD film S12 $\left([\mathrm{B}]_{\text {SIMS }}>10^{21} \mathrm{~cm}^{-3}\right)$, almost no photon energy dependence is observed. The fitting data shown in Table II reveal a reduction of the pseudogap $E_{\Delta \pi}$ with increase in boron content. Graphitization of the NCD films could explain the reduction of the pseudogap $E_{\Delta \pi}$. Raman spectroscopy, however, did not provide a clear evidence for changes of the $D$ and $G$ bands' intensity and position with increasing boron concentration (Fig. 2). In fact, the intensity ratio $I_{\text {diamond }} /\left(I_{D}+I_{G}\right)$ (Fig. 3) was almost independent on the doping level. On the other hand, the contribution of boron acceptors to the optical absorption in the low energy range of the spectrum could result into an apparent reduction of the pseudogap $E_{\Delta \pi}$. Thus, we can conclude that the influence of the boron acceptor on the absorption coefficient at low energies range is very important, in good agreement with the photocurrent data.

Due to limitations of our PDS setup, we are not able to investigate the optical-absorption coefficient at energy higher than $4.25 \mathrm{eV}$. Taking into account the photocurrent data, transitions from $\pi$ states to the valence-band tail region and band tail to band tail transitions are expected to dominate the absorption processes at high energies, as in the case of the undoped PCD films. ${ }^{22}$ It is therefore, important to point out that calculated values of the optical-absorption coefficient are comparable to the values obtained by Achatz et al..$^{31}$ in nitrogen-free and nitrogen-doped UNCD films, confirming 
that the optical-absorption processes in nanocrystalline diamond films are dominated by the grain boundaries.

\section{CONCLUSIONS}

In this work we have studied the structural, electronic, and optical properties of boron-doped nanocrystalline diamond films. Raman spectroscopy was used to investigate the diamond quality of the films, confirming that the relative amount of amorphous carbon $(G$ and $D$ bands in Raman spectra) does not change significantly with increasing boron concentration. The electronic properties of B-doped NCD are controlled by the concentration of boron acceptors in the films. A change of the boron concentration by 5 orders of magnitude results in a change of conductivity of almost 10 orders of magnitude, from $1.5 \times 10^{-8} \Omega^{-1} \mathrm{~cm}^{-1}$ for nonintentionally boron-doped film $\left([\mathrm{B}]_{\text {SIMS }}=9.7 \times 10^{16} \mathrm{~cm}^{-3}\right)$ up to $76 \Omega^{-1} \mathrm{~cm}^{-1}$ for heavily $\mathrm{B}$ doped $\left([\mathrm{B}]_{\mathrm{SIMS}}=3.3\right.$ $\left.\times 10^{21} \mathrm{~cm}^{-3}\right)$. The carrier concentration for low and intermediate doped samples is thermally activated in the temperature range from 300 to $700 \mathrm{~K}$, with an activation energy decreasing with increasing boron concentration. The doping dependence of the activation energy was modeled using a quasiphenomenological relationship proposed by Pearson and Bardeen, ${ }^{45}$ which can result from the combination of different effects such as impurity level broadening and the electrostatic interaction between free carriers and ionized impurities. At higher boron doping $\left([\mathrm{B}]_{\text {SIMS }}>1.3 \times 10^{20} \mathrm{~cm}^{-3}\right)$, the conductivity and the carrier concentration show very weak temperature dependence and the samples exhibit metal-like properties, with the carrier concentration measured by Hall effect equal to the concentration of boron in the films. The mobility of the investigated NCD films decreases with increasing boron concentration, resembling the behavior expected for ionized impurity scattering: at low boron doping $\left([\mathrm{B}]_{\mathrm{SIMS}}=2.7 \times 10^{19} \mathrm{~cm}^{-3}\right)$ the mobility is $2.1 \mathrm{~cm}^{2} \mathrm{~V}^{-1} \mathrm{~s}^{-1}$, while at high boron doping $\left([\mathrm{B}]_{\mathrm{SIMS}}=3.3 \times 10^{21} \mathrm{~cm}^{-3}\right)$ the mobility decreases down to $0.24 \mathrm{~cm}^{2} \mathrm{~V}^{-1} \mathrm{~s}^{-1}$. The contribution of different conduction mechanisms-valence band, hopping, and metal-like transport-has been discussed. While valence-band transport is suggested to dominate at low boron concentrations and high temperature, hopping transport between acceptor states occurs at higher doping levels and at low temperature. The metal-insulator transition of NCD films occurs for boron concentrations above 3 $\times 10^{20} \mathrm{~cm}^{-3}$, similarly to the case of single crystalline and polycrystalline diamonds. The influence of the grain boundaries has been carefully discussed. In contrast to poly- and nanocrystalline Si films, charge trapping at grain boundaries does not play the same dominant role as discussed by Seto. ${ }^{40}$ However, the lower value of the carrier mobility measured in B-doped NCD as compared to polycrystalline and single crystalline diamonds suggests that scattering at the grain boundaries is not negligible.

The optical characterization confirmed the strong influence of the grain boundaries on the optical properties of NCD films. The results of spectrally resolved photocurrent and photothermal deflection spectroscopy in the low energy range, between 0.5 and $1.0 \mathrm{eV}$, confirmed that boron as well as $s p^{2}$ carbon phases in the grain boundaries govern the optical-absorption process. We conclude that while the electronic properties are mainly governed by the addition of boron impurities, the optical properties are mainly determined by the presence of grain boundaries.

\section{ACKNOWLEDGMENTS}

This work was supported by the EC Research Training Network "DRIVE" under Contract No. RTN-CT-2004512224, by the Research Foundation-Flanders (FWO) under Research Programs No. G.0068.07 and No. G.0430.07, and the IAP-P6/42 project "Quantum Effects in Clusters and Nanowires.'
${ }^{1}$ B. Bi, W.-S. Huang, J. Asmussen, and B. Golding, Diamond Relat. Mater. 11, 677 (2002).

${ }^{2}$ F. Bénédic, M. B. Assouar, F. Mohasseb, O. Elmazria, P. Alnot, and A. Gicquel, Diamond Relat. Mater. 13, 347 (2004).

${ }^{3}$ M. Dipalo, J. Kusterer, K. Janischowsky, and E. Kohn, Phys. Status Solidi A 203, 3036 (2006).

${ }^{4}$ M. Kubovic, K. Janischowsky, and E. Kohn, Diamond Relat. Mater. 14, 514 (2005).

${ }^{5}$ S. Mitura, A. Mitura, P. Niedzielski, and P. Couvrat, Chaos, Solitons Fractals 10, 2165 (1999).

${ }^{6}$ P. Hollman, O. Wänstrand, and S. Hogmark, Diamond Relat. Mater. 7, 1471 (1998).

${ }^{7}$ P. S. Siew, K. P. Loh, W. C. Poh, and H. Zhang, Diamond Relat. Mater. 14, 426 (2005).

${ }^{8}$ P. Christiaens, V. Vermeeren, S. Wenmackers, M. Daenen, K. Haenen, M. Nesládek, M. van de Ven, M. Ameloot, L. Michiels, and P. Wagner, Biosens. Bioelectron. 22, 170 (2006).

${ }^{9}$ S. Q. Lud, M. Steenackers, R. Jordan, P. Bruno, D. M. Gruen, P.
Feulner, J. A. Garrido, and M. Stutzmann, J. Am. Chem. Soc. 128, 16884 (2006).

${ }^{10}$ B. Hong, Joungchel Lee, R. W. Collins, Y. Kuang, W. Drawl, R. Messier, T. T. Tsong, and Y. E. Strausser, Diamond Relat. Mater. 6, 55 (1997).

${ }^{11}$ T.-S. Yang, J.-Y. Lai, C.-L. Cheng, and M.-S. Wong, Diamond Relat. Mater. 10, 2161 (2001).

${ }^{12}$ M. Daenen, O. A. Williams, J. D'Haen, K. Haenen, and M. Nesládek, Phys. Status Solidi A 203, 3005 (2006).

${ }^{13}$ W. Kulisch, C. Popov, V. Vorlicek, P. N. Gibson, and G. Favaro, Thin Solid Films 515, 1005 (2006).

${ }^{14}$ O. A. Williams, M. Daenen, J. D’Haen, K. Haenen, J. Maes, V. V. Moshchalkov, M. Nesladek, and D. M. Gruen, Diamond Relat. Mater. 15, 654 (2006).

${ }^{15}$ P. Achatz, O. A. Williams, P. Bruno, D. M. Gruen, J. A. Garrido, and M. Stutzmann, Phys. Rev. B 74, 155429 (2006).

${ }^{16}$ O. A. Williams, S. Curat, J. E. Gerbi, and D. M. Gruen, Appl. Phys. Lett. 85, 1680 (2004). 
${ }^{17}$ E. P. Visser, G. J. Bauhuis, G. Janssen, W. Vollenberg, W. J. P. van Enckevort, and L. J. Giling, J. Phys.: Condens. Matter 4, 7365 (1992).

${ }^{18}$ T. H. Borst and O. Weiss, Phys. Status Solidi A 154, 423 (1996).

${ }^{19}$ J.-P. Lagrange, A. Deneuville, and E. Gheeraert, Diamond Relat. Mater. 7, 1390 (1998).

${ }^{20}$ J.-P. Lagrange, A. Deneuville, and E. Gheeraert, Carbon 37, 807 (1999).

${ }^{21}$ R. Locher, J. Wagner, F. Fuchs, M. Maier, P. Gonon, and P. Koidl, Diamond Relat. Mater. 4, 678 (1995).

${ }^{22}$ C. E. Nebel, Semicond. Sci. Technol. 18, S1 (2003).

${ }^{23}$ E. Bustarret, E. Gheeraert, and K. Watanabe, Phys. Status Solidi A 199, 9 (2003).

${ }^{24}$ T. Klein, P. Achatz, J. Kacmarcik, C. Marcenat, F. Gustafsson, J. Marcus, E. Bustarret, J. Pernot, F. Omnes, Bo E. Sernelius, C. Persson, A. Ferreira da Silva, and C. Cytermann, Phys. Rev. B 75, 165313 (2007).

${ }^{25}$ E. Gheeraert, A. Deneuville, and J. Mambou, Diamond Relat. Mater. 7, 1509 (1998).

${ }^{26}$ K. Ushizawa, K. Watanabe, T. Ando, I. Sakaguchi, M. NishitaniGamo, Y. Sato, and H. Kanda, Diamond Relat. Mater. 7, 1719 (1998).

${ }^{27}$ J. A. N. Gonçalves, G. M. Sandonato, and K. Iha, Diamond Relat. Mater. 11, 1578 (2002).

${ }^{28}$ M. Nesladek, L. M. Stals, A. Stesmans, K. Iakoubovskij, G. J. Adriaenssens, J. Rosa, and M. Vanecek, Appl. Phys. Lett. 72, 3306 (1998).

${ }^{29}$ R. Kravets, V. Ogorodniks, A. Poruba, P. Moravec, M. Nesladek, J. Rosa, and M. Vanecek, Phys. Status Solidi A 193, 502 (2002).

${ }^{30}$ M. Vanecek, R. Kravets, A. Poruba, J. Rosa, M. Nesladek, and S. Koizumi, Diamond Relat. Mater. 12, 521 (2003).

${ }^{31}$ P. Achatz, J. A. Garrido, M. Stutzmann, O. A. Williams, D. M. Gruen, A. Kromka, and D. Steinmüller, Appl. Phys. Lett. 88, 101908 (2006).

${ }^{32}$ A. C. Ferrari and J. Robertson, Phys. Rev. B 63, 121405(R) (2001).

${ }^{33}$ H. Kuzmany, R. Pfeiffer, N. Salk, and B. Guenther, Carbon 42, 911 (2004)

${ }^{34}$ S. Prawer and R. J. Nemanich, Philos. Trans. R. Soc. London, Ser. A 362, 2537 (2004).

${ }^{35}$ P. Gonon, E. Gheeraert, A. Deneuville, F. Fontaine, L. Abello, and G. Locazeau, J. Appl. Phys. 78, 7059 (1995).

${ }^{36}$ F. Pruvost and A. Deneuville, Diamond Relat. Mater. 10, 531
(2001).

${ }^{37}$ J. W. Ager III, W. Walukiewicz, M. McCluskey, M. A. Plano, and M. I. Lansdtrass, Appl. Phys. Lett. 66, 616 (1995).

${ }^{38}$ M. Nesladek, D. Tromson, C. Mer, P. Bergonzo, P. Hubik, and J. J. Mares, Appl. Phys. Lett. 88, 232111 (2006).

${ }^{39}$ R. J. Zhang, S. T. Lee, and Y. W. Lam, Diamond Relat. Mater. 5, 1288 (1996).

${ }^{40}$ J. Y. N. Seto, J. Appl. Phys. 46, 5247 (1975).

${ }^{41}$ O. A. Williams, O. Douhéret, M. Daenen, K. Haenen, E. Ōsawa, and M. Takahashi, Chem. Phys. Lett. 445, 255 (2007).

${ }^{42}$ S. Ghodbane and A. Deneuville, Diamond Relat. Mater. 15, 589 (2006)

${ }^{43}$ P. V. Zinin, I. Kudryashov, N. Konishi, L. C. Ming, V. L. Solozhenko, and S. K. Sharma, Spectrochim. Acta, Part A 61, 2386 (2005).

${ }^{44}$ K. Thonke, Semicond. Sci. Technol. 18, S20 (2003).

${ }^{45}$ G. L. Pearson and J. Bardeen, Phys. Rev. 75, 865 (1949).

${ }^{46}$ T. F. Lee and T. C. McGill, J. Appl. Phys. 46, 373 (1975).

${ }^{47}$ D. M. Malta, J. A. von Windheim, H. A. Wynands, and B. A. Fox, J. Appl. Phys. 77, 1536 (1995).

${ }^{48}$ M. Werner, O. Dorsch, H. U. Baerwind, E. Obermeier, L. Haase, W. Seifert, A. Ringhandt, C. Johnston, S. Romani, H. Bishop, and P. R. Chalker, Appl. Phys. Lett. 64, 595 (1994).

${ }^{49}$ B. Massarani, J. C. Bourgoin, and R. M. Chrenko, Phys. Rev. B 17, 1758 (1978).

${ }^{50}$ S. M. Sze, Physics of Semiconductors, 2nd ed. (Wiley, New York, 1981).

${ }^{51}$ J. J. Mares, P. Hubık, M. Nesladek, D. Kindl, and J. Kristofik, Diamond Relat. Mater. 15, 1863 (2006).

${ }^{52}$ J. J. Mares, M. Nesladek, P. Hubık, D. Kindl, and J. Kristofik, Diamond Relat. Mater. 16, 1 (2007).

${ }^{53}$ J. J. Mares, M. Nesladek, P. Hubık, and J. Kristofik, Diamond Relat. Mater. 16, 921 (2007).

${ }^{54}$ E. Bustarret, P. Achatz, B. Sacépé, C. Chapelier, C. Marcenat, L. Ortga, and T. Klein, Philos. Trans. R. Soc. London, Ser. A $\mathbf{3 6 6}$, 267 (2008).

${ }^{55}$ E. Bustarret, Phys. Status Solidi A 205, 997 (2008).

${ }^{56}$ R. Kravets, K. Johnston, J. Potmesil, V. Vorlicek, and M. Vanecek, Diamond Relat. Mater. 15, 559 (2006).

${ }^{57}$ M. Nesladek, M. Vanecek, and L. M. Stals, Phys. Status Solidi A 154, 283 (1996); M. Nesladek, K. Meykens, L. M. Stals, M. Vanecek, and J. Rosa, Phys. Rev. B 54, 5552 (1996). 